Draft Version August 10, 2018

Preprint typeset using LTTEX style emulateapj v. 01/23/15

\title{
ON VERTICALLY GLOBAL, HORIZONTALLY LOCAL MODELS FOR ASTROPHYSICAL DISKS
}

\author{
Colin P. McNally and Martin E. Pessah \\ Niels Bohr International Academy, Niels Bohr Institute, Blegdamsvej 17, DK-2100, Copenhagen Ø, Denmark; cmcnally@nbi.dk, mpessah@nbi.dk \\ Draft version August 10, 2018
}

\begin{abstract}
Disks with a barotropic equilibrium structure, for which the pressure is only a function of the density, rotate on cylinders in the presence of a gravitational potential, so that the angular frequency of such a disk is independent of height. Such disks with barotropic equilibria can be approximately modeled using the shearing box framework, representing a small disk volume with height-independent angular frequency. If the disk is in baroclinic equilibrium, the angular frequency does in general depend on height and it is thus necessary to go beyond the standard shearing box approach. In this paper, we show that given a global disk model, it is possible to develop approximate models that are local in horizontal planes without an expansion in height with shearing-periodic boundary conditions. We refer to the resulting framework as the vertically global shearing box (VGSB). These models can be non-axisymmetric for globally barotropic equilibria but should be axisymmetric for globally baroclinic equilibria. We provide explicit equations for this VGSB which can be implemented in standard magnetohydrodynamic codes by generalizing the shearing-periodic boundary conditions to allow for a height-dependent angular frequency and shear rate. We also discuss the limitations that result from the radial approximations that are needed in order to impose height-dependent shearing periodic boundary conditions. We illustrate the potential of this framework by studying a vertical shear instability and examining the modes associated with the magnetorotational instability.
\end{abstract}

Keywords: accretion, accretion disks — instabilities — ISM: structure - magnetic fields - magnetohydrodynamics (MHD) - plasmas

\section{INTRODUCTION}

Astrophysical disks play a crucial role in the formation, evolution, and fate of a wide variety of celestial objects, by mediating the transport of mass, energy, and angular momentum. Building realistic disk models is of fundamental importance for understanding, for example, protoplanetary disks around young stars, accretion flows onto stellar compact objects and active galactic nuclei, as well as the interstellar medium in galactic disks. The large dynamic range involved makes it particularly challenging to produce detailed global numerical simulations of these systems. Moreover, while global models allow us to investigate large-scale phenomena, local models with a hierarchy of increasingly complex microphysics have proved critical to elucidating the processes that are crucial at small scales. Because of this, several types of local approximations have been employed for studying astrophysical disks.

Hill (1878) pioneered the use of a local approximation to study the dynamics of particles orbiting a host system and subject to encounters with a perturber, and used it to study the motion of the Moon. Spitzer \& Schwarzschild (1953) and Goldreich \& Lynden-Bell (1965) applied these ideas to galactic disks using the concept of a locally shearing coordinate system. This approach constitutes the basis of the shearing sheet framework which has been widely used to study the dynamics of orbiting particles and planetesimals, as well as local processes in hydrodynamic and magnetohydrodynamic disks. The implementation of the concept behind the shearing sheet, with an appropriate shear-periodic radial-boundary condition (Wisdom \& Tremaine 1988), forms a computational model used for studying local disk dynamics referred to as the shear- ing box (Brandenburg et al. 1995, Hawley et al. 1995).

Shearing box models solve the equations of motion for the fluid in a local cartesian frame co-rotating with the disk at a fiducial radius. In the standard framework, the differential rotation of the disk is locally accounted for with a heightindependent angular frequency. This is appropriate for disks with a barotropic equilibrium for which the pressure is only a function of density and thus rotate on cylinders. The shearing box framework relies on a first order expansion of the steady bulk flow in the radial direction, which is the highest order compatible with shearing periodic boundary conditions. Depending on whether zeroth or first order expansions are considered for the gravitational field in the direction perpendicular to the disk, usually denoted with the coordinate $z$, this leads to the so called unstratified (Hawley et al. 1995, 1996) or stratified (Brandenburg et al. 1995, Stone et al. 1996) shearing box models. This stratified, compressible shearing box is what we will refer to in this paper as the standard shearing box (SSB). These approximations are appropriate when the disk is thin and the vertical scales of interest are small compared to the fiducial disk radius. There have been works that retained the correct expression for the vertical component of the gravitational field (Matsuzaki et al. 1997; Korpi et al. 1999), allowing for larger vertical domains to be considered. However, this generalization does not allow to study of disks with baroclinic equilibria without relaxing the assumption that the angular frequency is height-independent. In the early formulation of the shearing sheet, Goldreich \& Lynden-Bell (1965) avoided making any approximation in height $(z)$, which is possible when considering barotropic equilibrium structures.

Astrophysical disks with baroclinic equilibrium structure, for which the pressure is not solely a function of density, 
posses angular frequency profiles that depend in general on height, especially if these are not thin. Therefore, building a framework to study these disks demands going beyond the SSB, where the assumption that the angular frequency is height-independent is rooted deep. In this work, we generalize the SSB by considering the full height-dependence of a steady state, axisymmetric bulk flow to leading order in radius, without making any expansion in the vertical coordinate. We show that given a global disk model, it is possible to develop approximate models that are local in horizontal planes and global in height and are amenable to shearingperiodic boundary conditions. These models can be nonaxisymmetric for disks with a barotropic global equilibrium but should be axisymmetric for disks with a baroclinic global equilibrium. We term the resulting framework the vertically global shearing box (VGSB). The terminology of "vertically global' specifically and solely refers to the approach of never making a expansion in the vertical direction in the derivation. This yields a model which allows us to account for the vertical variation of gravity without approximations and the possible presence of vertical shear. As we discuss below, the radial expansions that are needed to apply height-dependent shearing periodic boundary conditions do limit the range of vertical scales which can be modeled approximately. In spite of its limitations, the VGSB formalism provides a novel framework that goes beyond the SSB and promises to provide a bridge between strictly local and fully global approaches to model astrophysical disks.

The paper is organized as follows. We derive the equations involved in the VGSB framework in Section 3, providing some of the algebraic details in Appendix A. We state the final form of the VGSB equations and discuss its novel features in Section 4 For convenience, we provide a self-contained summary of the VGSB equations that can be incorporated in magnetohydrodynamic codes in Appendix B. We use this new framework to explore the behavior of two important instabilities in a baroclinic context. We demonstrate that a linear vertical shear instability (VSI), akin to those studied by Goldreich \& Schubert (1967); Fricke (1968) and Nelson et al. (2013), appears in the VGSB in Section 5 We examine some basic aspects of the magnetorotational instability (MRI) in the VGSB in Section 6. We conclude by briefly discussing the limitations and several potential applications of the VGSB in Section 7. In addition, in Appendix Cwe outline some details related to neglecting curvature terms, in Appendix D we analyze the hydrodynamic momentum equation in order to assess under what conditions it is acceptable to discard radial pressure gradients in the SSB and VGSB, and in Appendix E discuss issues related to potential vorticity in shearing boxes.

\section{EQUATIONS OF MOTION}

We are concerned with the equations of ideal magnetohydrodynamics, in cylindrical coordinates $(r, \phi, z)$, in a refer- ence frame rotating with angular frequency $\Omega_{\mathrm{F}}=\Omega_{\mathrm{F}} \hat{\boldsymbol{z}}$, i.e.,

$$
\begin{aligned}
\frac{\partial \rho}{\partial t}+\nabla \cdot(\rho \boldsymbol{v}) & =0 \\
\frac{\partial \boldsymbol{v}}{\partial t} & +(\boldsymbol{v} \cdot \nabla) \boldsymbol{v}=\Omega_{\mathrm{F}}^{2} r \hat{\boldsymbol{r}}-2 \Omega_{\mathrm{F}} \times \boldsymbol{v} \\
& -\nabla \Phi-\frac{\nabla P}{\rho}+\frac{1}{\rho} \boldsymbol{J} \times \boldsymbol{B} \\
\frac{\partial \boldsymbol{B}}{\partial t} & =\nabla \times(\boldsymbol{v} \times \boldsymbol{B}) \\
\frac{\partial e}{\partial t}+\nabla \cdot(e \boldsymbol{v}) & =-P(\nabla \cdot \boldsymbol{v})
\end{aligned}
$$

Here, $\rho$ is the mass density, $v$ is the fluid velocity in the rotating frame, $\boldsymbol{B}$ is the magnetic field, with $\nabla \cdot \boldsymbol{B}=0$, e is the internal energy density, $P(\rho, e)$ is the pressure determined through an equation of state, and $\Phi(r, z)$ is the gravitational potential, which is assumed to be cylindrically symmetric, but not necessarily spherical. The current density is $\boldsymbol{J} \equiv \nabla \times \boldsymbol{B} / \mu_{0}$, with $\mu_{0}$ a constant dependent on the unit system adopted.

Fluid flows described by these equations are subject to conservation laws. It is thus important to understand under what circumstances these properties are satisfied by the equations describing the local dynamics involving expansions of the original set of equations. It is easy to show that the approximations embodied in the standard isothermal shearing box are such that the vortex lines of an inviscid flow are frozen into the fluid (Kelvin's Circulation Theorem) and that the magnetic flux is also frozen into the fluid in the absence of magnetic dissipation (Alfvén's Frozen-in Theorem).

Understanding under what conditions these properties also hold for the equations of motion that result from invoking a local approximation of global disk models which have a baroclinic equilibrium structure is more subtle. Here, we state the general versions of the aforementioned theorems in order to prepare the ground to address these issues in subsequent sections. These conservation theorems can be derived by calculating the Lagrangian derivative of the fluxes associated with the vorticity and the magnetic field. It is thus useful to recall that, see e.g., (Blackman 2013), any vector field $\boldsymbol{Q}$ satisfies

$$
\begin{aligned}
\left(\frac{\partial}{\partial t}\right. & +\boldsymbol{v} \cdot \nabla) \int_{\mathrm{S}} \boldsymbol{Q} \cdot \boldsymbol{d} \boldsymbol{S} \\
& =\int_{\mathrm{S}}\left[\frac{\partial \boldsymbol{Q}}{\partial t}-\nabla \times(\boldsymbol{v} \times \boldsymbol{Q})+\boldsymbol{v}(\nabla \cdot \boldsymbol{Q})\right] \cdot \boldsymbol{d} \boldsymbol{S},
\end{aligned}
$$

where the integral is carried out over any open surface $\mathrm{S}$ advected by the flow with velocity $\boldsymbol{v}$.

\subsection{Kelvin's Circulation Theorem}

The momentum equation (2) for an an inviscid, unmagnetized, barotropic flow in the rotating frame is given by

$$
\left(\partial_{t}+\boldsymbol{v} \cdot \nabla\right) \boldsymbol{v}=\Omega_{\mathrm{F}}^{2} r \hat{\boldsymbol{r}}-2 \Omega_{\mathrm{F}} \times \boldsymbol{v}-\nabla \Theta,
$$

where $\Theta=\Phi+h$ is the generalized gravito-thermal potential, where $h$ is the enthalpy, with $d h=d P / \rho$. The equation governing the evolution of the vorticity is thus

$$
\partial_{t}(\nabla \times \boldsymbol{v})=\nabla \times\left[\boldsymbol{v} \times\left(\nabla \times \boldsymbol{v}+2 \boldsymbol{\Omega}_{\mathrm{F}}\right)\right],
$$


which preserves the solenoidal character of the vorticity. This implies that, by virtue of Equation (5) with $\boldsymbol{Q}=\nabla \times \boldsymbol{v}+2 \boldsymbol{\Omega}_{\mathrm{F}}$, vortex lines are frozen into the fluid, i.e., the flow preserves the circulation $\Gamma$

$$
\left(\partial_{t}+\boldsymbol{v} \cdot \nabla\right) \Gamma=0
$$

with

$$
\Gamma \equiv \int_{\mathrm{S}}\left(\nabla \times \boldsymbol{v}+2 \boldsymbol{\Omega}_{\mathrm{F}}\right) \cdot \boldsymbol{d} \boldsymbol{S}=\oint_{\mathrm{L}}\left(\boldsymbol{v}+\boldsymbol{\Omega}_{\mathrm{F}} \times \boldsymbol{r}\right) \cdot d \boldsymbol{l},
$$

where $\mathrm{L}$ is a closed contour, delimiting the open surface $\mathrm{S}$, advected by the flow with velocity $\boldsymbol{v}$.

\subsection{Alfvén's Frozen-in Theorem}

The induction equation (3) preserves the divergence of the magnetic field, i.e.,

$$
\left(\partial_{t}+\boldsymbol{v} \cdot \nabla\right)(\nabla \cdot \boldsymbol{B})=0 .
$$

This implies that, provided that $\nabla \cdot \boldsymbol{B}=0$ at some initial time, the magnetic flux remains frozen into the fluid, i.e.,

$$
\left(\partial_{t}+\boldsymbol{v} \cdot \nabla\right) \Phi_{\mathrm{B}}=0
$$

with

$$
\Phi_{\mathrm{B}}=\int_{\mathrm{S}} \boldsymbol{B} \cdot \boldsymbol{d} \boldsymbol{S}
$$

where the integral is carried out over any open surface $\mathrm{S}$ advected by the flow with velocity $\boldsymbol{v}$. This follows from Equation (5) with $\boldsymbol{Q}=\boldsymbol{B}$ and the induction equation (3).

\section{THE VERTICALLY GLOBAL, HORIZONTALLY LOCAL APPROXIMATION}

We seek to derive a set of equations that describe the local dynamics of the magnetized fluid with respect to a known steady state bulk flow around a point co-rotating with the disk at a distance $r_{0}$. Here, we outline the steps of the derivation, which is carried out in detail below.

1. We find a suitable steady flow and background equilibrium, which enables the derivation of exact equations of motion for the departures from this solution.

2. We transform to a locally cartesian coordinate system. We expand the bulk flow and background equilibrium to leading order in the radial direction leaving the direction perpendicular to the disk midplane unaltered.

3. We determine under which circumstances the resulting equations are amenable to being solved with shearingperiodic boundary conditions, which could depend on height for disk models with baroclinic global equilibria.

We show explicitly these steps for the momentum and induction equations, while we state the results for the continuity equation and energy equation that are simpler to work with.

\subsection{Equations for Departures from Background Equilibrium}

\subsubsection{Bulk Flow and Background Equilibrium}

As in the SSB, we first seek a steady background flow. In this case, we will not make any a priori assumptions about the $z$-dependence of the angular frequency.

We begin by noting that the momentum equation (2) admits a force-free (magnetic fields playing no role), steady state solution $\boldsymbol{V} \equiv V(r, z) \hat{\boldsymbol{\phi}}$, with

$$
V(r, z)=r\left[\Omega(r, z)-\Omega_{\mathrm{F}}\right],
$$

where the angular frequency is

$$
\Omega^{2}(r, z) \equiv \frac{1}{r \rho_{h}} \frac{\partial P\left(\rho_{h}, e_{h}\right)}{\partial r}+\frac{1}{r} \frac{\partial \Phi}{\partial r},
$$

and the vertical, hydrostatic pressure gradient satisfies

$$
\frac{1}{\rho_{h}} \frac{\partial P\left(\rho_{h}, e_{h}\right)}{\partial z}=-\frac{\partial \Phi}{\partial z} .
$$

Here, $\rho_{h}(r, z)$ and $e_{h}(r, z)$ are the mass density and energy density associated with the steady state background flow.

\subsubsection{Momentum and Induction Equations}

Using Equations (14) and $(15)$ we can recast the gravitational force in the momentum equation (2) in terms of the angular frequency and the pressure gradient, both corresponding to the steady state bulk flow. We obtain

$$
\begin{gathered}
\left(\frac{\partial}{\partial t}+\boldsymbol{v} \cdot \nabla\right) \boldsymbol{v}=\left[\Omega_{\mathrm{F}}^{2}-\Omega^{2}(r, z)\right] r \hat{\boldsymbol{r}}-2 \boldsymbol{\Omega}_{\mathrm{F}} \times \boldsymbol{v} \\
+\frac{\nabla P\left(\rho_{h}, e_{h}\right)}{\rho_{h}}-\frac{\nabla P(\rho, e)}{\rho}+\frac{1}{\rho} \boldsymbol{J} \times \boldsymbol{B} .
\end{gathered}
$$

The velocity field describing the departure from the bulk flow satisfying Equations $(13)-(15)$

$$
\boldsymbol{w} \equiv \boldsymbol{v}-V(r, z) \hat{\boldsymbol{\phi}}
$$

evolves according to the momentum equation 1

$$
\begin{aligned}
\frac{\partial \boldsymbol{w}}{\partial t} & +\left[\Omega(r, z)-\Omega_{\mathrm{F}}\right]\left(\frac{\partial w_{r}}{\partial \phi} \hat{\boldsymbol{r}}+\frac{\partial w_{\phi}}{\partial \phi} \hat{\boldsymbol{\phi}}+\frac{\partial w_{z}}{\partial \phi} \hat{\boldsymbol{z}}\right)+\boldsymbol{w} \cdot \nabla \boldsymbol{w} \\
& +w_{r} r \frac{\partial \Omega(r, z)}{\partial r} \hat{\boldsymbol{\phi}}+w_{z} \frac{\partial r \Omega(r, z)}{\partial z} \hat{\boldsymbol{\phi}}+2 \boldsymbol{\Omega}(r, z) \times \boldsymbol{w} \\
& =\frac{\nabla P\left(\rho_{h}, e_{h}\right)}{\rho_{h}}-\frac{\nabla P(\rho, e)}{\rho}+\frac{1}{\rho} \boldsymbol{J} \times \boldsymbol{B} .
\end{aligned}
$$

This equation is exact and it displays the particular feature that the last term on the left-hand side resembles the Coriolis acceleration, with one important difference. The angular frequency involved is not the fixed angular frequency of the rotating frame, $\Omega_{\mathrm{F}}$, but rather the angular frequency of the steady state flow, $\boldsymbol{\Omega}(r, z)$. As a quick check, note that if $P(\rho, e)=P\left(\rho_{h}, e_{h}\right)$ and $\boldsymbol{J} \times \boldsymbol{B}=0$ then there are no departures from the steady state bulk flow, i.e., $\boldsymbol{w}=0$.

Taking the induction equation (3) and replacing the velocity

\footnotetext{
1 We provide the algebraic details of this derivation in Appendix A
} 

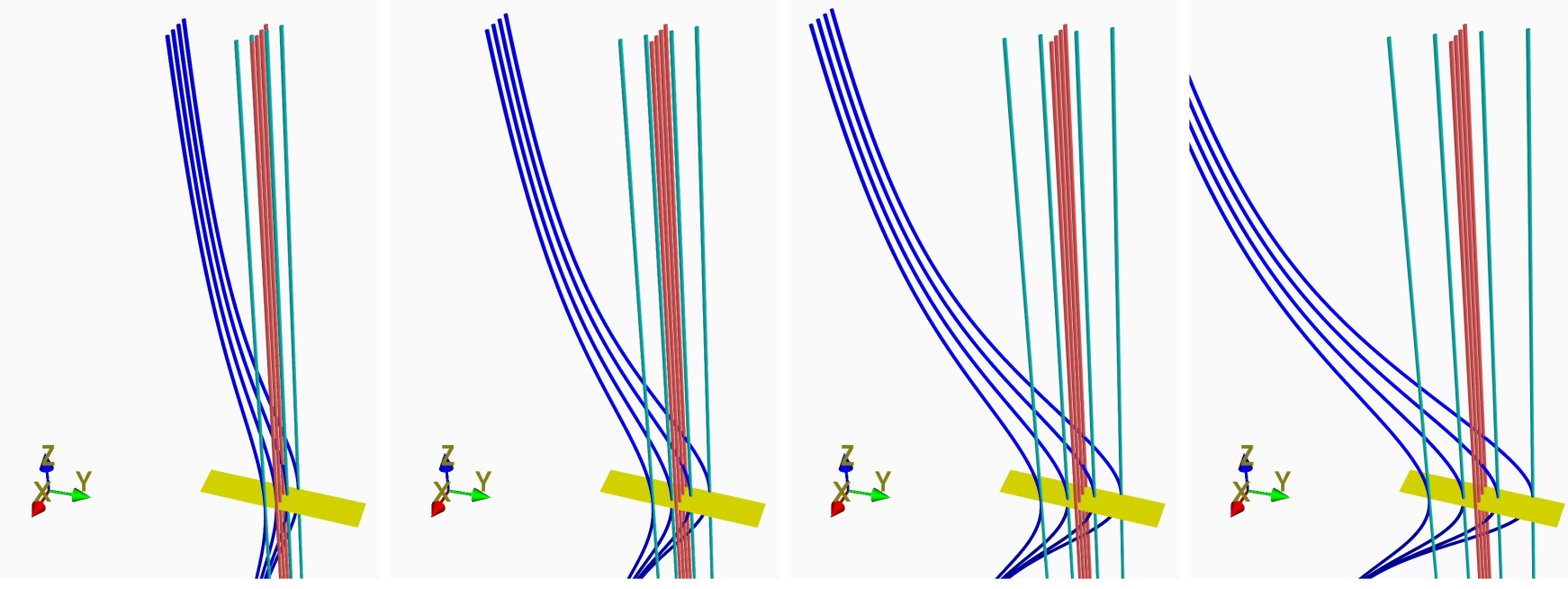

Figure 1. Rendering of Lagrangian tracer lines initially placed at $y=0$ in the steady state flow of the VGSB and standard shearing box. The VGSB provides a horizontally local, vertically global representation of a baroclinic equilibrium disk model with a cylindrical temperature dependence, which is described in more detail in Section 4.3.1 with a sound speed to rotation speed ratio of $c_{\mathrm{s} 0} / v_{\mathrm{K} 0}=0.1, p=-1.5$, and $q=-1$. The standard shearing box shown is for a Keplerian thin disk. The panels corresponds to $t \Omega_{0}(0)=0.5,1.0,1.5,2.0$, from left to right. The yellow surface marks the midplane of the shearing box and the top of the lines is at $z=3 r_{0}$. Red: initial position of tracer lines. Blue: evolution of tracer lines in the VGSB following the flow $\boldsymbol{V}(x, z)=\left[V_{0}(z)+S_{0}(z) x\right] \hat{\boldsymbol{y}}$ from Equations 82 - 83 . Green: evolution of tracer lines in the standard shearing box following the flow $\boldsymbol{V}(x, z)=-(3 / 2) \Omega_{0}(0) x \hat{\boldsymbol{y}}$.

field with $\boldsymbol{v} \equiv V(r, z) \hat{\boldsymbol{\phi}}+\boldsymbol{w}$ yields, after some algebra,

$$
\begin{aligned}
& \frac{\partial \boldsymbol{B}}{\partial t}+\left[\Omega(r, z)-\Omega_{\mathrm{F}}\right]\left(\frac{\partial B_{r}}{\partial \phi} \hat{\boldsymbol{r}}+\frac{\partial B_{\phi}}{\partial \phi} \hat{\boldsymbol{\phi}}+\frac{\partial B_{z}}{\partial \phi} \hat{\boldsymbol{z}}\right) \\
& =B_{r} r \frac{\partial \Omega(r, z)}{\partial r} \hat{\boldsymbol{\phi}}+B_{z} \frac{\partial r \Omega(r, z)}{\partial z} \hat{\boldsymbol{\phi}}+\nabla \times(\boldsymbol{w} \times \boldsymbol{B}) .
\end{aligned}
$$

\subsubsection{Background-flow Advection and Shear Rate}

The fact that the speed of the background flow $\boldsymbol{V} \equiv$ $V(r, z) \hat{\phi}$ depends, in general, on height implies that the departures from the bulk flow will be advected and sheared in a height-dependent way. This motivates the definition of the advection operator

$$
\mathcal{D} \equiv \frac{\partial}{\partial t}+\left[\Omega(r, z)-\Omega_{\mathrm{F}}\right] \frac{\partial}{\partial \phi},
$$

which is defined so that it acts on scalar fields, such as the density $\rho$, and on each of the components of a vector field, e.g., $\boldsymbol{w}$ and $\boldsymbol{B}$, but not on the unit coordinate-vectors, i.e., $\mathcal{D}\{\hat{\boldsymbol{r}}, \hat{\boldsymbol{\phi}}\}=0$. It is also convenient to define the shear rate

$$
S(r, z) \equiv r \frac{\partial \Omega(r, z)}{\partial r} .
$$

Using these definitions, Equations (18) and 19p become, without approximations,

$$
\begin{aligned}
(\mathcal{D} & +\boldsymbol{w} \cdot \nabla) \boldsymbol{w}=-2 \Omega(r, z) \hat{\boldsymbol{z}} \times \boldsymbol{w}-S(r, z) w_{r} \hat{\boldsymbol{\phi}} \\
& -w_{z} \frac{\partial V(r, z)}{\partial z} \hat{\boldsymbol{\phi}}+\frac{\nabla P\left(\rho_{h}, e_{h}\right)}{\rho_{h}}-\frac{\nabla P(\rho, e)}{\rho}+\frac{1}{\rho} \boldsymbol{J} \times \boldsymbol{B},
\end{aligned}
$$

$$
\mathcal{D} \boldsymbol{B}=S(r, z) B_{r} \hat{\boldsymbol{\phi}}+B_{z} \frac{\partial V(r, z)}{\partial z} \hat{\boldsymbol{\phi}}+\nabla \times(\boldsymbol{w} \times \boldsymbol{B}) .
$$

\subsection{Local Approximation in Horizontal Planes}

We now seek to derive a set of equations of motion which is local in radius and azimuth by expanding Equation (22) and (23) around a fiducial point $\boldsymbol{r}_{0}=\left(r_{0}, \phi_{0}, 0\right)$. In order to simplify this task, we choose a reference frame that corotates with the bulk flow at radius $r_{0}$, i.e.,

$$
\Omega_{\mathrm{F}}=\Omega\left(r_{0}, 0\right) .
$$

We also adopt a coordinate frame centered at $\boldsymbol{r}_{0}$ with locally cartesian coordinates $\boldsymbol{x}=(x, y, z)$, such that $x=r-r_{0}$ and $y \equiv r_{0}\left(\phi-\phi_{0}\right)$, with $x / r_{0} \ll 1$ and $y / r_{0} \ll 1$. In this locally cartesian frame, the differential vector operators are well approximated by their cartesian versions, provided that the radial coordinate versor $\hat{\boldsymbol{r}}(\phi) \approx \hat{\boldsymbol{r}}\left(\phi_{0}\right)^{2}$ Because of the axisymmetric character of the background flow, in what follows, we choose $\phi_{0}=0$ without loss of generality.

In this locally cartesian coordinate system, we can expand to leading order in $(x, y)$ the various functions appearing in the momentum and induction equations, Equation (22) and (23), respectively.

\subsubsection{Approximation of the Bulk Flow}

The local approximations of the angular frequency, the bulk flow in Equation (13), and the advection operator in Equation 20 yield

$$
\begin{aligned}
\Omega(x, z) & \equiv \Omega_{0}(z)+\left.\frac{\partial \Omega(r, z)}{\partial r}\right|_{r=r_{0}} x, \\
V(x, z) & \equiv V_{0}(z)+S_{0}(z) x, \\
\mathcal{D}_{0} & \equiv \frac{\partial}{\partial t}+\left[V_{0}(z)+S_{0}(z) x\right] \frac{\partial}{\partial y} .
\end{aligned}
$$

\footnotetext{
${ }^{2}$ This approximation, which is valid when the physical extent in horizontal planes is small, leads to neglecting terms related to the curvilinear character of the cylindrical coordinate system originally chosen to describe the bulk flow as $\boldsymbol{V} \equiv V(r, z) \hat{\boldsymbol{\phi}}$. See Appendix C for more details.
} 
Here, we have defined the local, height-dependent angular frequency, bulk flow, and shear rate, all evaluated at the fiducial radius $r_{0}$, i.e.,

$$
\begin{aligned}
\Omega_{0}(z) & \equiv \Omega\left(r_{0}, z\right), \\
V_{0}(z) & \equiv V\left(r_{0}, z\right), \\
S_{0}(z) & \left.\equiv r_{0} \frac{\partial \Omega(r, z)}{\partial r}\right|_{r=r_{0}} .
\end{aligned}
$$

The operator $\mathcal{D}_{0}$ is a height-dependent generalization of the shearing sheet advection operator originally introduced in Spitzer \& Schwarzschild (1953); Goldreich \& Lynden-Bell (1965). In order to illustrate the action of the advection operator $\mathcal{D}_{0}$, Figure 1 shows the effects of considering the local bulk flow in Equation (26) that results from expanding Equation (13) to leading order in the radial direction, leaving unaltered the vertical dependence. In this particular example, we have considered a baroclinic equilibrium global disk model with a cylindrical temperature structure, which is discussed in detail in Section 4.3.1

\subsubsection{Approximation of Momentum and Induction Equations}

Using the approximations above, we arrive at expressions for the momentum and induction equations, Equations (22) and (23), which are correct to leading order in $x / r_{0}$ and $y / r_{0}$ :

$$
\begin{gathered}
\left(\mathcal{D}_{0}+\boldsymbol{w} \cdot \nabla\right) \boldsymbol{w}=-2 \Omega_{0}(z) \hat{\boldsymbol{z}} \times \boldsymbol{w}-S_{0}(z) w_{x} \hat{\boldsymbol{y}} \\
-w_{z} \frac{\partial V(x, z)}{\partial z} \hat{\boldsymbol{y}}+\frac{\nabla P\left(\rho_{h}, e_{h}\right)}{\rho_{h}}-\frac{\nabla P(\rho, e)}{\rho}+\frac{1}{\rho} \boldsymbol{J} \times \boldsymbol{B}, \\
\mathcal{D}_{0} \boldsymbol{B}=S_{0}(z) B_{x} \hat{\boldsymbol{y}}+B_{z} \frac{\partial V(x, z)}{\partial z} \hat{\boldsymbol{y}}+\nabla \times(\boldsymbol{w} \times \boldsymbol{B}) .
\end{gathered}
$$

Here, all the differential operators are defined in a cartesian coordinate system centered at the fiducial radius $r_{0}$.

Because we have retained the leading order in all the approximations involving the bulk-flow, the two important flow properties discussed in Section 2 remain unaltered. In the case of an inviscid, unmagnetized, barotropic flow the momentum equation (31) that results from the local approximation in horizontal planes leads to

$$
\left(\mathcal{D}_{0}+\boldsymbol{w} \cdot \nabla\right) \Gamma=0 .
$$

Furthermore, the induction equation (32) that results from the local approximation in horizontal planes preserves the solenoidal character of the the magnetic field, i.e.,

$$
\left(\mathcal{D}_{0}+\boldsymbol{w} \cdot \nabla\right)(\nabla \cdot \boldsymbol{B})=0 .
$$

This implies that the magnetic flux is frozen into the fluid flow that results from the local expansion in horizontal planes, i.e.,

$$
\left(\mathcal{D}_{0}+\boldsymbol{w} \cdot \nabla\right) \Phi_{\mathrm{B}}=0 .
$$

Therefore, the local approximation in horizontal planes leads to equations that still satisfy Kelvin's circulation theorem and Alfvén's frozen in theorem discussed in Section 2

\subsection{Compatibility of the Local Approximation with Shearing-periodic Boundary Conditions}

\subsubsection{Shearing-periodic Boundary Conditions in the SSB}

All the explicit coordinate dependences in the equations of motion defining the SSB are contained in the advection operator

$$
\mathcal{D}_{0}^{\mathrm{SSB}} \equiv \frac{\partial}{\partial t}+x S_{0}(0) \frac{\partial}{\partial y},
$$

which is obtained as a limit of the operator $\mathcal{D}_{0}$ introduced in Equation (27). The explicit dependence on the coordinate $x$ can be eliminated by the linear transformation $t^{\prime}=t, x^{\prime}=x$, $z^{\prime}=z$, and

$$
y^{\prime}=y-x S_{0}(0) t .
$$

In the primed coordinate system, the advection operator simply becomes $\mathcal{D}_{0}^{\mathrm{SSB}}=\partial_{t}^{\prime}$, and the equations of motion can be solved by using strictly periodic boundary conditions in horizontal planes, i.e.,

$$
\begin{aligned}
& f\left(x^{\prime}, y^{\prime}, z^{\prime}, t^{\prime}\right)=f\left(x^{\prime}+L_{x}, y^{\prime}, z^{\prime}, t^{\prime}\right), \\
& f\left(x^{\prime}, y^{\prime}, z^{\prime}, t^{\prime}\right)=f\left(x^{\prime}, y^{\prime}+L_{y}, z^{\prime}, t^{\prime}\right),
\end{aligned}
$$

and appropriate boundary conditions for the vertical boundaries. Note that in the original coordinate system, the equations for the departures from the bulk flow satisfy shearingperiodic boundary conditions given by

$$
\begin{aligned}
& f(x, y, z, t)=f\left(x+L_{x}, y+S_{0}(0) L_{x} t, z, t\right), \\
& f(x, y, z, t)=f\left(x, y+L_{y}, z, t\right) .
\end{aligned}
$$

\subsubsection{Height-dependent Shearing-periodic Boundary Conditions}

Defining an approximate, radially local set of equations and boundary conditions for disks with baroclinic equilibria, for which the angular frequency is in general a function of height, is more subtle.

The coordinate dependence arising through the advection operator $\mathcal{D}_{0}$ in Equation (27) can still be removed by defining the linear transformation $t^{\prime}=t, x^{\prime}=x, z^{\prime}=z$, and

$$
y^{\prime}=y-\left[V_{0}(z)+S_{0}(z) x\right] t .
$$

In this primed coordinate system, the advection operator is coordinate-independent, i.e., $\mathcal{D}_{0}=\partial_{t}^{\prime}$. Therefore, if this were the only explicit coordinate dependence then, in each horizontal plane, it would be enough to consider the heightdependent, shearing-periodic boundary conditions given by

$$
\begin{aligned}
& f(x, y, z, t)=f\left(x+L_{x}, y+S_{0}(z) L_{x} t, z, t\right), \\
& f(x, y, z, t)=f\left(x, y+L_{y}, z, t\right) .
\end{aligned}
$$

However, the coordinate dependence induced by the terms proportional to

$$
\frac{\partial V(x, z)}{\partial z}=\frac{\partial V_{0}(z)}{\partial z}+x \frac{\partial S_{0}(z)}{\partial z}
$$

on the right-hand sides of Equations (31) and (32) cannot be eliminated by the same coordinate transformation that removes the $x$-dependence in $\mathcal{D}_{0}$. This prevents Equation (31) and (32) from being solved with the shearing-periodic boundary conditions in Equation (41) in a straightforward way.

In what follows we analyze the consequences of proceeding by neglecting the term proportional to the coordinate $x$ in 
Equation (42). We thus approximate this equation as

$$
\frac{\partial V(x, z)}{\partial z} \simeq \frac{\partial V_{0}(z)}{\partial z}
$$

With the exception of the background hydrostatic profile which will be dealt with in the next section, this approximation eliminates the explicit coordinate dependence on the right-hand sides of Equations (31) and (32), leading to

$$
\begin{aligned}
& \left(\mathcal{D}_{0}+\boldsymbol{w} \cdot \nabla\right) \boldsymbol{w}=-2 \Omega_{0}(z) \hat{\boldsymbol{z}} \times \boldsymbol{w}-S_{0}(z) w_{x} \hat{\boldsymbol{y}} \\
& -w_{z} \frac{\partial V_{0}(z)}{\partial z} \hat{\boldsymbol{y}}+\frac{\nabla P\left(\rho_{h}, e_{h}\right)}{\rho_{h}}-\frac{\nabla P(\rho, e)}{\rho}+\frac{1}{\rho} \boldsymbol{J} \times \boldsymbol{B},
\end{aligned}
$$

$$
\mathcal{D}_{0} \boldsymbol{B}=S_{0}(z) B_{x} \hat{\boldsymbol{y}}+B_{z} \frac{\partial V_{0}(z)}{\partial z} \hat{\boldsymbol{y}}+\nabla \times(\boldsymbol{w} \times \boldsymbol{B}) .
$$

Because the only coordinate dependences arise through the advection operator $\mathcal{D}_{0}$, these equations are compatible with the height-dependent, shearing-periodic boundary conditions Equations (41). By reducing the coordinate dependence down to the advection operator $\mathcal{D}_{0}$, and the background equilibrium $\nabla P\left(\rho_{h}, e_{h}\right) / \rho_{h}$ we have brought these equation closer to compatibility with with the height-dependent, shearingperiodic boundary conditions Equations (41). The background equilibrium will be treated in Section 3.3.5 but first we deal with issues which arise from this step. The approximation embodied in Equation (43) does, in general, affect the validity of the conservation theorems discussed in Section 2 We proceed by showing that Equations (44) and (45) do satisfy Kelvin's circulation theorem and Alfven's frozen-in theorem when the underlying global disk model has a barotropic equilibrium or when we consider axisymmetry.

\subsubsection{Radially local, Vertically Global Hydrodynamic Disk Models}

It can be seen that equation (44) when applied to an inviscid, barotropic, unmagnetized flow, leads to

$$
\left(\mathcal{D}_{0}+\boldsymbol{w} \cdot \nabla\right) \Gamma=0
$$

This means that under the conditions over which Kelvin's circulation theorem is satisfied, the approximation embodied in Equation 43 does not lead to spurious sources of circulation when considering Equation (31) instead of (44).

If the global disk model under consideration has baroclinic equilibrium, the circulation $\Gamma$ is no longer conserved and thus, for physical reasons, $\left(\mathcal{D}_{0}+\boldsymbol{w} \cdot \nabla\right) \Gamma$ is no longer expected to vanish. However, one should also realize that the approximation invoked in Equation (43) leads to a source term that contributes spuriously to the evolution of the circulation

$$
\left(\mathcal{D}_{0}+\boldsymbol{w} \cdot \nabla\right) \Gamma=\int_{\mathbf{S}}\left[\nabla \times\left(x w_{z} \frac{\partial S_{0}(z)}{\partial z} \hat{\boldsymbol{y}}\right)\right] \cdot \boldsymbol{d} \boldsymbol{S}+\ldots
$$

Here, the dots represent the physical sources of circulation present in fluids which are either viscous or baroclinic. The spurious source of circulation in Equation (47) vanishes under axisymmetry. In order to demonstrate this, let us examine the integral involved. Using Stoke's theorem, it follows that

$$
\int_{\mathbf{S}}\left[\nabla \times\left(x w_{z} \frac{\partial S_{0}(z)}{\partial z} \hat{\boldsymbol{y}}\right)\right] \cdot \boldsymbol{d} \boldsymbol{S}=\oint_{\mathbf{L}}\left(x w_{z} \frac{\partial S_{0}(z)}{\partial z}\right) \hat{\boldsymbol{y}} \cdot d \boldsymbol{l} .
$$

In axisymmetry, i.e., $\partial_{y}=0$, the problem reduces to understanding the dynamics of the fluid in the $(x, z)$ plane. Under this condition, the line integral over a closed loop vanishes.

Thus, given a global hydrodynamic disk model, corresponding to either barotropic or baroclinic equilibria, Equation (44) can be used to define an associated disk model that is local in horizontal planes but global in height. This model can be non-axisymmetric for disks with globally barotropic equilibria but should be axisymmetric for disks with globally baroclinic equilibria.

The flow of hydrodynamic fluids is constrained by the evolution of the potential vorticity as governed by Ertel's theorem (see, e.g. Pedlosky 1982). In Appendix E we derive the equations associated with the evolution of potential vorticity in the framework of the VGSB and discuss how these relate to the Kelvin circulation theorem alluded to in this section. The details of this also depend on the final approximations needed to make the model compatible with shearing periodic radial boundaries in Section 3.3.5. Before that, we discuss the analogous issue which occurs with magnetic fields in the induction equation.

\subsubsection{Radially local, Vertically Global MHD Disk Models}

Let us now consider the implications for the induction equation. In general, neglecting the term proportional to the coordinate $x$ in Equation (43) leads to an approximated induction equation that no longer preserves the solenoidal character of the magnetic field. More specifically, Equation (45) leads to

$$
\left(\mathcal{D}_{0}+\boldsymbol{w} \cdot \nabla\right)(\nabla \cdot \boldsymbol{B})=-x \frac{\partial S_{0}(z)}{\partial z} \frac{\partial B_{z}}{\partial y} .
$$

Taken at face value, this implies that Equation (43) induces spurious generation of magnetic monopoles that will break flux freezing even in the absence of dissipation. Therefore, in the case of a magnetized fluid, while eliminating the explicit $x$-dependence that can not be removed by a coordinate transformation allows the induction equation to be solved with shearing-periodic, height-dependent boundary conditions, this approximation, in general, would destroy the solenoidal character of the magnetic field.

However, the spurious evolution of the divergence of the magnetic field that results from the approximation Equation (43) is absent in Equation (49) if the underlying global disk model is has a barotropic equilibrium or axisymmetry, i.e., $\partial_{y}=0$, is considered.

Therefore, given a global disk model, it is possible to develop models which preserve the solenoidal character of the magnetic field that are local in horizontal planes and global in height that are amenable to shearing-periodic boundary conditions. These models can be non-axisymmetric for disks with globally barotropic equilibria but should be axisymmetric for disks with globally baroclinic equilibria. Under either of these conditions, the approximation invoked in Equation (43), which is necessary to employ the shearing-periodic boundary conditions given by Equation (41), does not lead 
to spurious source terms that could affect the evolution of the circulation, magnetic flux freezing, or the solenoidal character of the magnetic field, i.e.,

$$
\begin{aligned}
& \left(\mathcal{D}_{0}+\boldsymbol{w} \cdot \nabla\right) \Gamma=0, \\
& \left(\mathcal{D}_{0}+\boldsymbol{w} \cdot \nabla\right) \Phi_{\mathrm{B}}=0,
\end{aligned}
$$

and

$$
\left(\mathcal{D}_{0}+\boldsymbol{w} \cdot \nabla\right)(\nabla \cdot \boldsymbol{B})=0
$$

\subsubsection{Approximation of the Background Equilibrium}

The fourth term on the right-hand side of Equation $(22)$ can be dealt with along the lines proposed by Goldreich \& Lynden-Bell (1965) for the hydrostatic background quantities

$$
\begin{aligned}
& \rho_{h}(r, z)=\rho_{h 0}(z)+\mathrm{O}\left(\frac{x}{r_{0}}\right), \\
& e_{h}(r, z)=e_{h 0}(z)+\mathrm{O}\left(\frac{x}{r_{0}}\right),
\end{aligned}
$$

where $\rho_{h 0}(z) \equiv \rho_{h}\left(r_{0}, z\right)$ and $e_{h 0}(z) \equiv e_{h}\left(r_{0}, z\right)$ are the leading order terms associated with the mass and internal energy density profiles of the background flow at the fiducial radiu ${ }^{3}$ Within the level of approximation we are working at, we thus have

$$
\frac{\nabla P\left(\rho_{h}, e_{h}\right)}{\rho_{h}}=\frac{\nabla P\left(\rho_{h 0}, e_{h 0}\right)}{\rho_{h 0}}+\mathrm{O}\left(\frac{x}{r_{0}}\right),
$$

where the vertical acceleration induced by the background pressure gradient is balanced by gravity at the fiducial radius

$$
\frac{1}{\rho_{h 0}} \frac{\partial P\left(\rho_{h 0}, e_{h 0}\right)}{\partial z}=-\frac{\partial \Phi_{0}(z)}{\partial z} \hat{\boldsymbol{z}}
$$

with $\Phi_{0}(z) \equiv \Phi\left(r_{0}, z\right)$, the gravitational potential evaluated at the fiducial radius $r_{0}$.

The set of approximations to the background described above can also be considered on more formal grounds by introducing a set of dimensionless parameters that describe the relative scale of the phenomena of interest and the departures from a thin, Keplerian disk. In Appendix D, we provide the details involved in this procedure, emphasizing in particular the handling of the terms related to pressure gradients in the momentum equation. Our considerations build on, and extend, the analysis carried out in Umurhan \& Regev (2004). The analysis suggests the condition $z<\sqrt{x r_{0}}$ as a limit on the height of the best modeled part of the domain. This criterion also applies to the SSB, as we demonstrate in Appendix $\mathrm{D}$

The set of equations that result from considering the approximations described in this section are compatible with the height-dependent, shearing-periodic boundary conditions Equations 41. This motivates introducing the VGSB.

\section{THE VERTICALLY GLOBAL SHEARING BOX (VGSB)}

\subsection{Equations of Motion for the VGSB}

\footnotetext{
${ }^{3}$ In the case of a barotropic equation of state, where $e=e(\rho)$ and thus $P(\rho, e)=P(\rho)$, only the expansion in Equation 53 is required, as in Goldreich \& Lynden-Bell (1965 Section 4).
}

Following the steps outlined in the previous section, we arrive at the expressions for the continuity, momentum, induction, and energy equations that define the framework of the vertically global shearing box (VGSB)

$$
\mathcal{D}_{0} \rho+\nabla \cdot(\rho \boldsymbol{w})=0
$$

$$
\begin{aligned}
&\left(\mathcal{D}_{0}+\boldsymbol{w} \cdot \nabla\right) \boldsymbol{w}+w_{z} \frac{\partial V_{0}(z)}{\partial z} \hat{\boldsymbol{y}} \\
&=-2 \Omega_{0}(z) \hat{\boldsymbol{z}} \times \boldsymbol{w}-S_{0}(z) w_{x} \hat{\boldsymbol{y}} \\
&-\frac{\nabla P}{\rho}-\frac{\partial \Phi_{0}(z)}{\partial z} \hat{\boldsymbol{z}}+\frac{1}{\rho} \boldsymbol{J} \times \boldsymbol{B}, \\
&\left(\mathcal{D}_{0}+\boldsymbol{w} \cdot \nabla\right) \boldsymbol{B}-B_{z} \frac{\partial V_{0}(z)}{\partial z} \hat{\boldsymbol{y}} \\
&=S_{0}(z) B_{x} \hat{\boldsymbol{y}}+(\boldsymbol{B} \cdot \nabla) \boldsymbol{w}-\boldsymbol{B}(\nabla \cdot \boldsymbol{w}), \\
& \mathcal{D}_{0} e+\nabla \cdot(e \boldsymbol{w})=-P(\nabla \cdot \boldsymbol{w}) .
\end{aligned}
$$

Here, all the operators are defined in a cartesian coordinate system centered at the fiducial radius $r_{0}$.

The only explicit coordinate dependence in the VGSB arises through the advection operator $\mathcal{D}_{0}$, which is linear in the coordinate $x$ for all heights $z$. This implies that the equations are suitable for being solved with a vertically varying shear-periodic $x$-direction boundary condition. The radial and azimuthal boundary conditions for mapping a field variable $f$ in a VGSB of size $L_{x} \times L_{y} \times L_{z}$ are, respectively ${ }^{4}$

$$
\begin{aligned}
& f(x, y, z, t)=f\left(x+L_{x}, y+S_{0}(z) L_{x} t, z, t\right), \\
& f(x, y, z, t)=f\left(x, y+L_{y}, z, t\right) .
\end{aligned}
$$

In order to completely define the problem, appropriate boundary conditions in the vertical direction must be specified.

The set of Equations $(57)-(60)$, together with the boundary conditions Equations (61)- 62) lead to approximate radially local, vertically global disk models if the underlying global disk model has a barotropic equilibrium or if we assume axisymmetry.

It is important to emphasize that the velocity $\boldsymbol{w}$ is the departure from the local approximation of the bulk flow and thus the total fluid velocity $\boldsymbol{v}$ in the VGSB is

$$
\boldsymbol{v}=\left[V_{0}(z)+S_{0}(z) x\right] \hat{\boldsymbol{y}}+\boldsymbol{w},
$$

where

$$
V_{0}(z)=r_{0}\left[\Omega_{0}(z)-\Omega_{0}(0)\right],
$$

and the local shear rate $S_{0}(z)$ is related to the generalization of the height-independent $q$-parameter in the SSB:

$$
q_{0}(z) \equiv-\frac{S_{0}(z)}{\Omega_{0}(0)} .
$$

At the midplane, i.e., $z=0$, the flow velocity $V(x, 0)=$ $S_{0}(0) x=-q_{0}(0) \Omega_{0}(0) x$ is the same as the steady state flow velocity in the SSB. However, in global disk models where the shear rate of the flow decreases to zero at high altitude, i.e., $S_{0}(z) \simeq 0$ and, therefore, $V_{0}(z) \simeq-r_{0} \Omega_{0}(0)$ for $z \gg r_{0}$. This is just the reflex motion induced by the rotating frame.

\footnotetext{
${ }^{4}$ The velocity $w_{y}$ is continuous across the shear-periodic $x$-boundary.
} 
This shows that in the limit $z \gg r_{0}$, Equations (57)- 60 reduce to the MHD equations in the absence of a gravitational field.

\subsection{Connecting the VGSB to the SSB}

In order to connect the SSB to the VGSB, with its generalization to baroclinic disk equilibria, it is useful to recall some results that apply to barotropic hydrostatic equilibria. If the pressure is $P=P(\rho)$ it follows that:

(i) The angular frequency $\Omega$ is independent of $z$. This can be derived by taking the curl of the momentum equation (6), which leads to

$$
r \frac{\partial \Omega^{2}}{\partial z}=0
$$

implying that barotropic equilibria rotate on cylinders $5^{5}$ i.e., $\Omega \equiv \Omega(r)$.

(ii) The generalized gravito-thermal potential $\Theta$ is independent of $z$. This follows from the vertical component of Equation (66), because the bulk flow velocity has only an an azimuthal component. This implies that

$$
\frac{\partial \Theta}{\partial z}=0
$$

and thus $\Theta \equiv \Theta(r)$, as stated.

(iii) The angular frequency can be obtained from the generalized gravito-thermal potential using the radial component of Equation (6) as

$$
r \Omega^{2}=\frac{\partial \Theta}{\partial r}
$$

Let us now focus our attention on a global isothermal disk in the gravitational potential of a point source of mass $M$, i.e.,

$$
\Phi(r, z)=\frac{-G M}{\sqrt{r^{2}+z^{2}}},
$$

with $G$ the gravitational constant. The pressure is $P=\rho c_{\mathrm{s} 0}^{2}$, with constant sound speed $c_{\mathrm{s} 0}$. Adopting a power-law density dependence with radius in the midplane, tha hydrostatic balance in the vertical direction implies that the density is

$\rho(r, z)=\rho_{0}\left(\frac{r}{r_{0}}\right)^{p} \exp \left[-\frac{v_{\mathrm{K}}^{2}(r)}{c_{\mathrm{s} 0}^{2}}\left(1-\frac{1}{\sqrt{1+(z / r)^{2}}}\right)\right]$,

where the constant $p \leq 0$ and we defined the Keplerian speed

$$
v_{\mathrm{K}}(r)=\sqrt{\frac{G M}{r}}
$$

In this case, the enthalpy is simply $h=c_{\mathrm{s} 0}^{2} \ln \rho$, and thus the generalized gravito-thermal potential $\Theta=\Phi+h$, is

$$
\Theta=c_{\mathrm{s} 0}^{2} \ln \left[\rho_{0}\left(\frac{r}{r_{0}}\right)^{p}\right]-v_{\mathrm{K}}^{2}(r) .
$$

\footnotetext{
5 This is often referred to as the Taylor-Proudman theorem, which is commonly credited to Taylor (1917); Proudman (1916). The same principle that we reference here, that barotropes rotate on cylinders, is also referred to in the literature as the Poincaré-Wavre therom (Tassoul 2000 Section 3.1.2) and the von Zeipel condition (von Zeipel 1924).
}

The angular frequency can be obtained from Equation $(68)$ as

$$
\Omega=\Omega_{\mathrm{K}}(r) \sqrt{1+p \frac{c_{\mathrm{s} 0}^{2}}{v_{\mathrm{K}}^{2}(r)}},
$$

where the Keplerian frequency is

$$
\Omega_{\mathrm{K}}(r)=\frac{v_{\mathrm{K}}(r)}{r}=\sqrt{\frac{G M}{r^{3}}} .
$$

The generalized gravito-thermal potential $\Theta$ and the angular frequency $\Omega$ are both independent of height, as expected.

Expanding Equations (70) and (73) in radius around $r_{0}$, we obtain expressions for the local values the angular frequency $\Omega_{0}$ and the shear rate $S_{0}$ that can be used, in the framework of the VGSB, to study isothermal disks which are not necessarily thin compared to the local radius $r_{0}$, i.e.,

$$
\begin{aligned}
\rho_{0}(z) & =\rho_{0} \exp \left[-\frac{v_{\mathrm{K} 0}^{2}}{c_{\mathrm{s} 0}^{2}}\left(1-\frac{1}{\sqrt{1+\left(z / r_{0}\right)^{2}}}\right)\right] \\
\Omega_{0} & =\Omega_{\mathrm{K} 0} \sqrt{1+p \frac{c_{\mathrm{s} 0}^{2}}{v_{\mathrm{K} 0}^{2}}} \\
S_{0} & =-\frac{3}{2} \Omega_{\mathrm{K} 0}\left(1+\frac{2 p}{3} \frac{c_{\mathrm{s} 0}^{2}}{v_{\mathrm{K} 0}^{2}}\right)\left(1+p \frac{c_{\mathrm{s} 0}^{2}}{v_{\mathrm{K} 0}^{2}}\right)^{-1 / 2}
\end{aligned}
$$

where we have defined $v_{\mathrm{K} 0} \equiv v_{\mathrm{K}}\left(r_{0}\right)$ and $\Omega_{\mathrm{K} 0} \equiv \Omega_{\mathrm{K} 0}\left(r_{0}\right)$.

These expressions are useful to show that in the limit of a cold, thin disk, i.e., $c_{\mathrm{S} 0} \ll v_{\mathrm{K} 0}$, the density profile becomes $\rho_{0}(z)=\exp \left[-\left(z / H_{0}\right)^{2} / 2\right]$, with $H_{0} / r_{0}=c_{\mathrm{s} 0} / v_{\mathrm{K} 0}$, and the angular frequency and shear rate become $\Omega_{0}=\Omega_{\mathrm{K} 0}$, $S_{0}=-3 / 2 \Omega_{\mathrm{K} 0}$, respectively, and Equations 57) 60 reduce to the equations for the SSB.

\subsection{Examples of VGSB Models for Global Equilibria}

We now consider two families of baroclinic, global disk equilibria that are characterized by angular frequencies and shear rates that depend on height, and are thus impossible to study within the standard shearing box approximation. The VGSB framework, embodied in Equations (57)-(59), together with their associated boundary conditions, can be used to produce axisymmetric, vertically global, horizontally local models for these astrophysical disks. After these examples, we summarize the new aspects of the baroclinic VGSB.

\subsubsection{VGSB for Global Disk Models with Cylindrical Temperature Structure}

There is a family of disk models which are isothermal in the vertical direction at any fiducial radius. These have been used, for example, by Nelson et al.(2013). The corresponding 

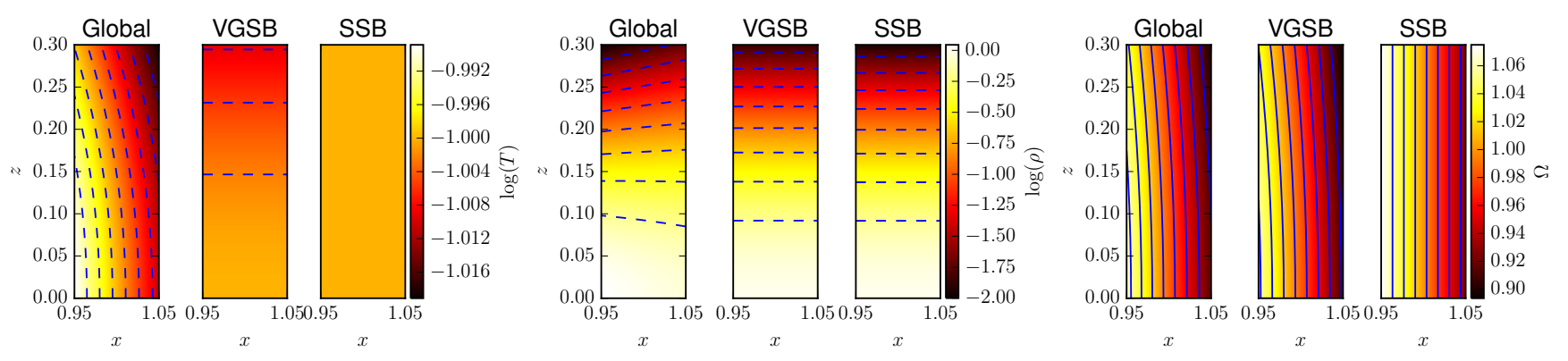

Figure 2. A global hydrostatic equilibrium with spherical temperature structure and local approximations. The analytical forms for the global and VGSB equilibrium are given in Section 4.3.2 with $\mu=3$ and $\nu=97$, yielding an aspect ratio of 0.1 . The three panels show, from left to right, the logarithm of temperature, logarithm of pressure, and angular velocity. Global: exact global equilibrium. VGSB: vertically global, horizontally local approximation. SSB: standard shearing box, horizontally and vertically local. In line with the analysis in Appendix D these plots show only the regions $z<\sqrt{x r_{0}}$.

global hydrostatic equilibrium configuration is given by

$$
\begin{aligned}
T(r) & \equiv T_{0}\left(\frac{r}{r_{0}}\right)^{q}, \\
\rho(r, z) & =\rho_{0}\left(\frac{r}{r_{0}}\right)^{p} \exp \left[-\frac{v_{\mathrm{K}}^{2}}{c_{\mathrm{s}}^{2}}\left(1-\frac{1}{\sqrt{1+(z / r)^{2}}}\right)\right] \\
\Omega(r, z) & =\Omega_{\mathrm{K}} \sqrt{1+(p+q) \frac{c_{\mathrm{s}}^{2}}{v_{\mathrm{K}}^{2}}+q\left(1-\frac{1}{\sqrt{1+(z / r)^{2}}}\right)},
\end{aligned}
$$

where $c_{\mathrm{S}}$ stands for the sound speed, assuming an ideal gas,

$$
c_{\mathrm{S}}^{2}(r)=c_{\mathrm{s} 0}^{2}\left(\frac{r}{r_{0}}\right)^{q}
$$

The local expansions involved in the VGSB for this global baroclinic equilibrium disk model are

$\Omega_{0}(z)=\Omega_{\mathrm{K} 0} \sqrt{1+(p+q) \frac{c_{\mathrm{s} 0}^{2}}{v_{\mathrm{K} 0}^{2}}+q\left(1-\frac{1}{\sqrt{1+\left(z / r_{0}\right)^{2}}}\right)}$,

$$
\begin{aligned}
& S_{0}(z)=-\frac{3}{2} \Omega_{\mathrm{K} 0} \\
& \times\left[1+\frac{(2-q)(p+q)}{3} \frac{c_{\mathrm{s} 0}^{2}}{v_{\mathrm{K} 0}^{2}}+q\left(1-\frac{1+(2 / 3)\left(z / r_{0}\right)^{2}}{\left[1+\left(z / r_{0}\right)^{2}\right]^{3 / 2}}\right)\right] \\
& \times\left[1+(p+q) \frac{c_{\mathrm{s} 0}^{2}}{v_{\mathrm{K} 0}^{2}}+q\left(1-\frac{1}{\sqrt{1+\left(z / r_{0}\right)^{2}}}\right)\right]^{-1 / 2}
\end{aligned}
$$

with $v_{\mathrm{K} 0}=v_{\mathrm{K}}\left(r_{0}\right)$ and $\Omega_{\mathrm{K} 0}=\Omega_{\mathrm{K} 0}\left(r_{0}\right)$.

Note that all the explicit $z$-dependences in $\Omega_{0}(z)$ and $S_{0}(z)$ arise only when $q \neq 0$. This is in agreement with the isothermal case, corresponding to $q=0$, in which the angular frequency is independent of height. It can also be seen from inspection of these equations that in the thin disk limit, i.e., $c_{\mathrm{S} 0} / v_{\mathrm{K} 0} \rightarrow 0$, the VGSB reduces to the $\mathrm{SSB}$ with $\Omega_{0}(0)=\Omega_{\mathrm{K} 0}, V_{0}(z)=0$, and $S_{0}(z)=-(3 / 2) \Omega_{\mathrm{K} 0}$, provided that $z \ll r_{0}$, even if the temperature depends on radius, i.e., $q \neq 0$. This is what ultimately justifies the use of a collection of SSBs to produce local barotropic equilibrium disk models, with height-independent angular frequency at different radii, even though the underlying global disk model has a baroclinic equilibrium structure.

\subsubsection{VGSB for Global Disk Models with Spherical Temperature Structure}

A spherical temperature dependence of the form

$$
T(r, z) \equiv T_{0} \frac{r_{0}}{\sqrt{r^{2}+z^{2}}},
$$

leads to a global hydrostatic disk configuration given by

$$
\begin{aligned}
& \rho(r, z)=\rho_{0}\left(\frac{r}{\sqrt{r^{2}+z^{2}}}\right)^{\nu}\left(\frac{\sqrt{r^{2}+z^{2}}}{r_{0}}\right)^{1-\mu}, \\
& \Omega(r, z)=\sqrt{\nu} \frac{c_{\mathrm{s} 0}}{r}\left(\frac{\sqrt{r^{2}+z^{2}}}{r_{0}}\right)^{-1 / 2} .
\end{aligned}
$$

Here, the power-law coefficients $\nu$ and $\mu$ are related via

$$
\nu+\mu=\frac{v_{\mathrm{K} 0}^{2}}{c_{\mathrm{S} 0}^{2}},
$$

and the sound speed is

$$
c_{\mathrm{s}}^{2}(r, z)=c_{\mathrm{s} 0}^{2} \frac{r_{0}}{\sqrt{r^{2}+z^{2}}},
$$

where $c_{\mathrm{S} 0}=c_{\mathrm{S}}\left(r_{0}, z\right)$ is the sound speed at the midplane at $r=r_{0}$. Such models have been used, for example, by Suzuki \& Inutsuka (2014).

The expansion of these expressions leads to the VGSB model corresponding to a global disk with a temperature structure varying in spherical shells. The local angular frequency and shear rate are, respectively,

$$
\begin{aligned}
\Omega_{0}(z)= & \Omega_{\mathrm{K} 0} \sqrt{\frac{\nu}{\nu+\mu}}\left[1+\left(\frac{z}{r_{0}}\right)^{2}\right]^{-1 / 4}, \\
S_{0}(z)=- & \Omega_{\mathrm{K} 0} \sqrt{\frac{\nu}{\nu+\mu}} \\
& \times\left[\frac{3}{2}+\left(\frac{z}{r_{0}}\right)^{2}\right]\left[1+\left(\frac{z}{r_{0}}\right)^{2}\right]^{-5 / 4} .
\end{aligned}
$$


In the limiting case in which $\mu=0$; the angular frequency and the shear rate take the Keplerian values at the midplane, i.e., $\Omega_{0}(0)=\Omega_{\mathrm{K} 0}$ and $S_{0}(0)=-(3 / 2) \Omega_{\mathrm{K} 0}$, and the parameter $\nu=v_{\mathrm{K} 0}^{2} / c_{\mathrm{s} 0}^{2}$ alone determines the disk thickness.

\subsubsection{New Aspects of the VGSB}

The approach that we followed in deriving the equations defining the framework of the VGSB is similar in spirit to the one employed in the derivation of the SSB. This consists of expanding the steady sate bulk flow, retaining only the leading order terms in the ratio $x / r_{0}$. The significant difference resides in that we have avoided making any expansion in the vertical direction. This allows us to retain the full heightdependence of the angular frequency. This is essential when dealing with disks in which the equilibrium structure is described by a baroclinic equation of state, since their angular frequency is in general a function of height.

In order to illustrate the new aspects that are open to examination by retaining the full height-dependence in Equations (25), 53, and (54), let us consider a baroclinic equilibrium global disk model, in which the temperature is a function of the spherical radius, as in Suzuki \& Inutsuka (2014). The four panels in Figure 2 compare the temperature, density, pressure, and angular frequency corresponding to the global equilibrium of the disk model (labeled "Global"), the local equilibrium defining the VGSB, and local equilibrium that would result in the SSB.

The VGSB provides a local representation of a global baroclinic equilibrium disk model, capturing effects that the SSB would not be able to account for. Because the lowest order discarded in Equation 25] is $\mathrm{O}\left(x^{2} / r_{0}^{2}\right)$, the radial dependence of the angular frequency is correct, to linear order in radius, for all heights ${ }^{6}$ Moreover, the VGSB retains the correct global vertical gradients in temperature, density, and pressure, while neglecting the local radial variation of the isopycnic, isothermal, isobaric surfaces. This is in sharp contrast with the local equilibrium involved in the SSB framework that is unable to capture the variation of the angular frequency with height, preventing its use when modeling disks with a baroclinic equilibrium structure. Note also that, for the case under consideration, the equilibrium density and pressure profiles involved in the standard isothermal shearing box are less accurate than the ones associated with the VGSB.

In the next two sections, we illustrate how the VGSB framework relates to, and extends, previous treatments of two disk instabilities that are relevant for a wide variety of astrophysical disks; namely the VSI, also known as the Goldreich-Schubert-Fricke (GSF) instability (Goldreich \& Schubert 1967; Fricke 1968), and the MRI (Balbus \& Hawley 1991). These instabilities will be the subject of future, more detailed, work.

\section{VSI IN THE VGSB}

Unmagnetized disks with shear profiles that depend on height have been long suspected to be unstable to various instabilities that feed off this angular frequency gradient.

\footnotetext{
6 Note that the radially local expansion removes the radial variation of temperature, resulting in a barotropic pressure-temperature relationship in the VGSB equilibrium, while the vertical shear of the global baroclinic equilibrium is retained.
}

The pioneering studies of Goldreich \& Schubert (1967) and Fricke (1968) invoked local approximations in both radius and height, capturing the essence of these instabilities but leaving open questions about their global behavior. These instabilities have been studied locally, and in conjunction with magnetorotational instability, in the context of accretion disks. It has been suggested that they can play a role in the low-conductivity regime characterizing protoplanetary disks (Urpin \& Brandenburg 1998, Rüdiger et al. 2002, Urpin 2003, Arlt \& Urpin 2004). The global, nonlinear evolution of these instabilities has ben recently studied in Nelson et al. (2013) by performing numerical simulations. This work includes an extension of the original local analysis, by considering the effects of compressibility and also an approximate, vertically global linear mode analysis. In this section, we show that the VGSB formalism recovers the local dispersion relation found in Nelson et al. (2013) and can be used to address their vertically global mode analysis without invoking the approximations related to compressibility considered by the authors $]^{7}$

There are general considerations which are common to both local and global approaches in height. Let us first write the VGSB equations for an unmagnetized disk for which the local angular frequency and shear rate derive from a global disk model with temperature $T(r)$ dependent on the cylindrical radius, as described in Section 4.3.1. Following Nelson et al. (2013), we make the change of variables $\Pi \equiv \log \rho$ so that Equations 57-60 become

$$
\begin{aligned}
& \mathcal{D}_{0} \Pi+\boldsymbol{w} \cdot \nabla \Pi+\nabla \cdot \boldsymbol{w}=0 \\
& \left(\mathcal{D}_{0}+\boldsymbol{w} \cdot \nabla\right) \boldsymbol{w}+w_{z} \frac{\partial V_{0}(z)}{\partial z} \hat{\boldsymbol{y}}= \\
& \quad-2 \Omega_{0}(z) \hat{\boldsymbol{z}} \times \boldsymbol{w}-S_{0}(z) w_{x} \hat{\boldsymbol{y}}-\frac{\nabla P}{\rho}-\frac{\partial \Phi_{0}(z)}{\partial z} \hat{\boldsymbol{z}}
\end{aligned}
$$

In order to derive the equations for the linear mode analysis that lead to the VSI starting from Equations (91) and (92), we proceed as follows: (i) we write the density variable as $\Pi=\Pi_{h}+\Pi_{f}$, i.e., the sum of its hydrostatic value and a fluctuation over this background, and the pressure as $P=c_{\mathrm{s}}^{2}\left(r_{0}, z\right) \rho$. (ii) We use the scales of length and time provided by $r_{0}$ and $\Omega_{0}(0)^{-1}$ to define the dimensionless variables $\boldsymbol{x} / r_{0} \rightarrow \boldsymbol{x}$ and $t \Omega_{0}(0) \rightarrow t$, so that $S_{0}(z) / \Omega_{0}(0) \rightarrow S_{0}(z)$ is the dimensionless shear rate, $c_{\mathrm{s} 0} / r_{0} \Omega_{0}(0) \rightarrow c_{\mathrm{S}}$ is the dimensionless sound speed, etc. (iii) We assume that all the perturbations are small and axisymmetric. (iv) We focus on radial scales that are small compared to the fiducial radius $r_{0}$ and take the Fourier transform of the set of equations (91) and (92), which reduces to making the substitution $f(x, z) \rightarrow \tilde{f}\left(k_{x}, z\right) \exp \left(\sigma t+i k_{x} x\right)$, for all flow variables, $\Pi_{f}, w_{x}, w_{y}$, and $w_{z}$, where the tilde denotes Fourier amplitudes. This procedure leads to the following set of dimension-

\footnotetext{
${ }^{7}$ After a preprint of this paper was first posted on the arXiv, several other works have treated the VSI, including Stoll \& Kley (2014); Barker \& Latter (2015); Lin \& Youdin (2015), and Umurhan et al. (2015). Notably Lin \& Youdin (2015) discuss the effects of fully removing the background radial pressure gradient, as done in this section.
} 


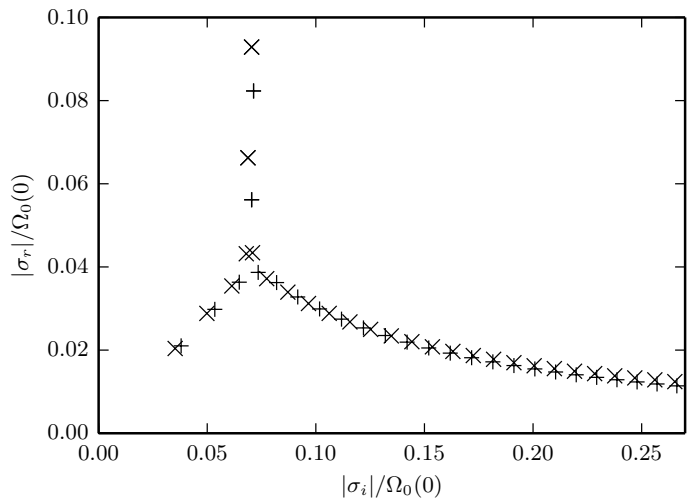

Figure 3. Comparison of the eigenvalues for the Nelson et al. (2013) approximation (their equation $39 \sqrt{8}$ ) and the fully compressible analysis given here for the parameters as listed in Nelson et al. (2013) (in our scaling $\left.k_{x}=+200 \pi, p=-1.5, q=-1, c_{\mathrm{s} 0} / v_{\mathrm{K}}=0.05,-5<z / H_{\mathrm{c}} \leq 5\right)$. To convert the values here to those scaling of Nelson et al. [2013) multiply by $2 \pi$. Symbols: + denotes our result, $\times$ denotes result calculated following Nelson et al. (2013). The main effect of including compressibility is to reduce the growth rate of the surface modes.

less equations

$$
\begin{aligned}
& \sigma \tilde{\Pi}_{f}=-\tilde{w}_{z} \partial_{z} \Pi_{h}-i k_{x} \tilde{w}_{x}-\partial_{z} \tilde{w}_{z} \\
& \sigma \tilde{w}_{x}=2 \Omega_{0}(z) \tilde{w}_{y}-i k_{x} c_{\mathrm{s}}^{2} \tilde{\Pi}_{f}, \\
& \sigma \tilde{w}_{y}=-\left[2 \Omega_{0}(z)+S_{0}(z)\right] \tilde{w}_{x}-\tilde{w}_{z} \partial_{z} V_{0}(z), \\
& \sigma \tilde{w}_{z}=-c_{\mathrm{s} 0}^{2} \partial_{z} \tilde{\Pi}_{f} .
\end{aligned}
$$

\subsection{Vertically Local Analysis of VSI}

Let us first show that the equations for the VGSB lead to the dispersion relation derived by Nelson et al. (2013, section 6.1), which is a generalization of the dispersion relation derived originally by GSF (Goldreich \& Schubert 1967; Fricke 1968), who considered an incompressible gas. In order to do this, we restrict the analysis to the vertical location about the height $z=z_{0}$, focusing on scales that are small in both the radial and vertical direction. Note that, in this case, the convenient time scale to define dimensionless variables is $\Omega_{0}\left(z_{0}\right)^{-1}$. Taking the Fourier transform of the set of equations (91) and (92) reduces to making the substitution $f(x, z) \rightarrow \tilde{f}\left(k_{x}, k_{z}\right) \exp \left(\sigma t+i k_{x} x+i k_{z} z\right)$, so that

$$
\begin{aligned}
\sigma \tilde{\Pi}_{f} & =\tilde{w}_{z} \frac{g}{c_{\mathrm{s} 0}^{2}}-i k_{x} \tilde{w}_{x}-i k_{z} \tilde{w}_{z}, \\
\sigma \tilde{w}_{x} & =2 \Omega_{0} \tilde{w}_{y}-i k_{x} c_{\mathrm{s} 0}^{2} \tilde{\Pi}_{f}, \\
\sigma \tilde{w}_{y} & =-\left(2 \Omega_{0}+S_{0}\right) \tilde{w}_{x}-\tilde{w}_{z} \partial_{z} V_{0}, \\
\sigma \tilde{w}_{z} & =-i k_{z} c_{\mathrm{s} 0}^{2} \tilde{\Pi}_{f},
\end{aligned}
$$

where the background quantities are understood to be evaluated at $z_{0}$ and we have used that $\partial_{z} \Pi_{h}=-g / c_{\mathrm{s} 0}^{2}$, where $g$ is the local value of the gravitational accelleration.

The characteristic polynomial of the homogeneous system of equations (97)- 100 yields the dispersion relation in terms of dimensionless variables

$$
\begin{aligned}
\sigma^{4} & +\sigma^{2}\left[c_{\mathrm{s} 0}^{2}\left(k_{x}^{2}+k_{z}^{2}\right)+i g k_{z}+2\left(S_{0}+2 \Omega_{0}\right) \Omega_{0}\right] \\
& +2\left(S_{0}+2 \Omega_{0}\right) \Omega_{0}\left(c_{\mathrm{s} 0}^{2} k_{z}^{2}+i k_{z} g\right)-2 \Omega_{0} c_{\mathrm{s} 0}^{2} k_{x} k_{z} \partial_{z} V_{0}=0 .
\end{aligned}
$$

This result is equivalent to the equation one before Equation (32) in Nelson et al. (2013). Therefore, the dispersion relation of the VSI present in global disk models with heightdependent angular frequencies is correctly obtained by the VGSB framework.

\subsection{Vertically Global Analysis of VSI}

In this section we perform a vertically global analysis of isothermal, axisymmetric unmagnetized perturbations by solving the coupled set of ordinary differential equations 93- 96. For a fixed radial wavenumber $k_{x} \gg 1$, the solution to the eigenvalue problem defined by these equations yields a set of eigenfunctions $\tilde{\Pi}_{f}, \tilde{w}_{x}, \tilde{w}_{y}$, and $\tilde{w}_{z}$ associated with the eigenvalues $\sigma$. We are particularly interested in finding the modes with growing amplitude, i.e., where the real part of the eigenvalue $\sigma_{r}>0$.

We compare the eigenvalues for a given set of parameters to those found through the approximated eigenproblem derived by Nelson et al. (2013), which invokes additional approximations. Reasonable agreement is found, as shown in Figure 3 . with the main difference being that the surface modes show slower growth when full compressibility is retained.

We illustrate the eigenmodes present in a disk model with cylindrical temperature structure $T(r)$, as presented in Section 4.3.1 in Figures 3 and 4 We consider as an example a global disk model with $p=-1.5, q=-1.0$, and the finite domain $-5 \leq z / H_{\mathrm{c}} \leq 5$, where $H_{\mathrm{c}} / r_{0}=c_{\mathrm{s} 0} / v_{\mathrm{K} 0}$ and $c_{\mathrm{S} 0} / v_{\mathrm{K} 0}=0.05$. These parameters match those presented in Nelson et al. (2013). We solve the problem corresponding to a single radial wavenumber $k_{x}= \pm 200 \pi$, associated to a wavelength of $10^{-2} r_{0}$, as follows. We discretize the problem in terms of Chebyshev cardinal functions on the Gauss-Lobatto grid, and the boundary condition $\tilde{w}_{z}=0$ is enforced by the "boundary bordering" method (Boyd 2000). We vary the resolution, using a maximum of 300 grid points (yielding a 1200 by 1200 matrix) to obtain the converged eigenvalues shown.

The basic pattern of modes shown in Figure 3 agrees well with the approximate analysis carried out by Nelson et al. (2013), who solved a second order differential equation that results from combining and approximate Equations $93-96$ 8 The important difference between these two approaches is that Nelson et al. (2013) make an approximation which removes full compressibility from the problem. We see that the fastest growing modes are relatively damped when full compressibility is retained in our analysis. In order to facilitate making a connection to their findings, we discuss the modes that we obtained using their terminology.

The eigenvalues have the symmetry in the complex plane $\sigma=\sigma_{r}+\operatorname{sign}\left(k_{x}\right)\left|\sigma_{i}\right|$. The fastest growing modes are a branch of "surface" modes with degenerate eigenvalues. In Figure 4 we show eigenfunctions where all components have been normalized by the complex value

$$
\tilde{\Pi}_{f}\left(-z_{m}\right)\left[\int_{-z_{m}}^{z_{m}}\left|\tilde{\Pi}_{f}(z) / \tilde{\Pi}_{f}\left(-z_{m}\right)\right|^{2} d z\right]^{1 / 2}
$$

where $z_{m}$ is the maximum $z$ of the domain. Because the Fourier amplitudes given by solving the eigenproblem as

\footnotetext{
${ }^{8}$ Note that Equation (39) of Nelson et al. (2013) contains a typo. It should have $-\sigma^{2} k^{2}$ as the final term according to their previous equation.
} 

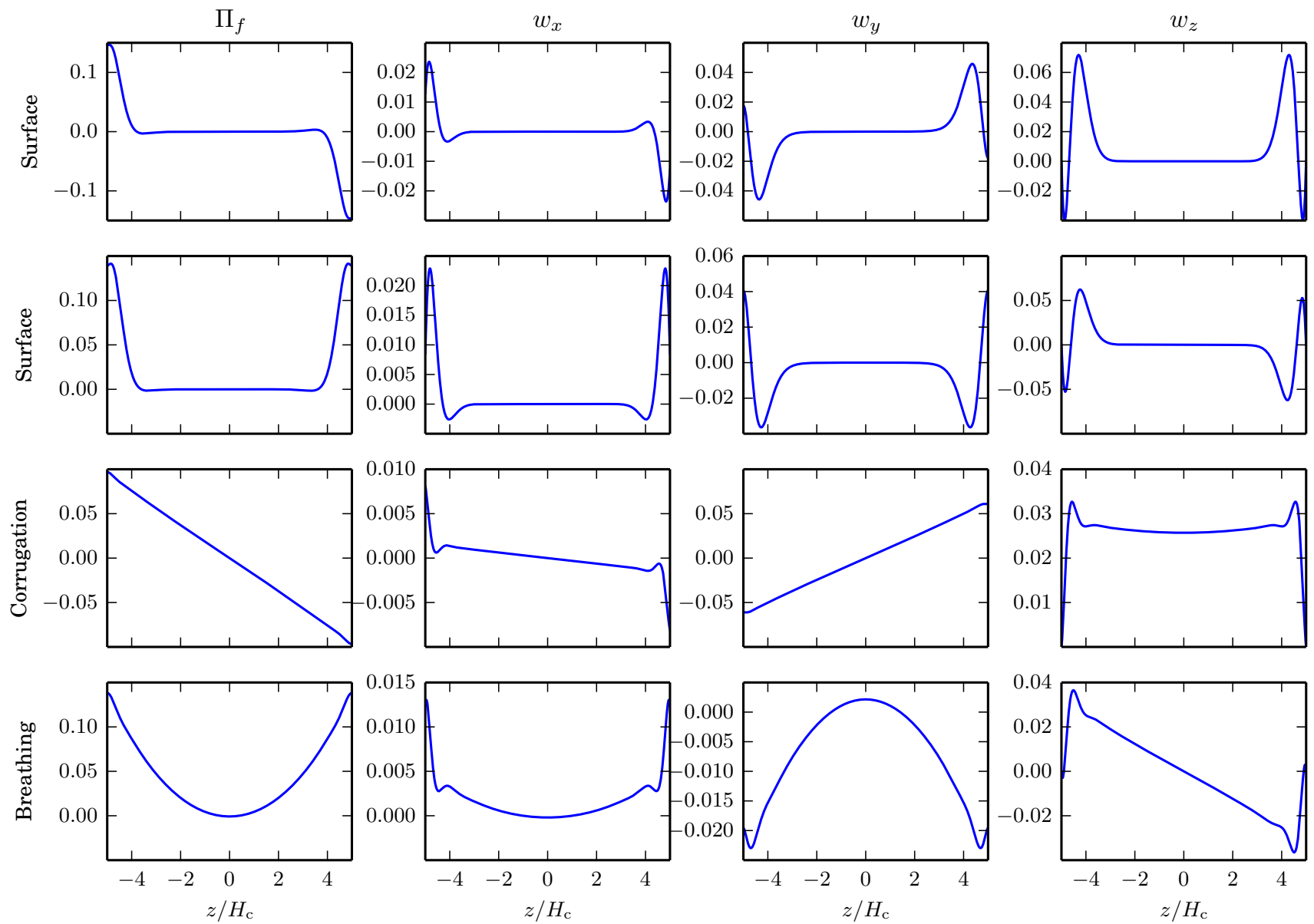

Figure 4. Four example eigenfunctions, transformed to real space, of the VSI, with normalization as given in text. Top Two Rows: the two degenerate fastest growing surface modes with degenerate eigenvalues, both modes have $\sigma \approx 0.0823 \pm 0.0713 i$. Third Row: fundamental corrugation mode with $\sigma \approx 0.0210 \pm$ $0.0380 i$. Fourth Row: fundamental breathing mode with $\sigma \approx 0.0298 \pm 0.0535 i$.

posed in transformed- $x$ Fourier space for $+k_{x}$ and $-k_{x}$ are complex conjugates, they correspond to a single real-valued eigenfunction when the $x$-direction Fourier transformation is inverted to bring the eigenfunction into real space. Therefore, we have plotted this real-valued eigenfunction resulting from the $+k_{x}$ and $-k_{x}$ pair. The first and second row in Figure 4 illustrate the fastest growing pair of modes with degenerate eigenvalue. A branch of eigenvalues proceeding from the origin of the complex plane contains the fundamental body modes, which are associated with "corrugation" and "breathing" modes. Like in Nelson et al. (2013), the eigenvalue closest to the origin is associated with the fundamental corrugation mode, shown in the third row of Figure 4. Next on this branch is the fundamental breathing mode, shown in the fourth row of Figure 4

Repeating the same calculation with a larger finite domain $-8 \leq z / H_{\mathrm{c}} \leq 8$, and thicker disk $c_{\mathrm{s} 0} / v_{\mathrm{K} 0}=0.1$ yields instead the modes shown in Figure 5. The remarkable change is the introduction if high frequency oscillations at high altitudes. The prodigious number of fast growing surface modes which appear in this calculation as the vertical size of the domain is increased suggests that magnetic fields will be a significant consideration in the astrophysical context for vertical shear instabilities. In the low density regime high above the disk midplane the gas will almost certainly be ionized in many types of astrophysical disks; the presence of magnetic fields ought then to have a significant impact on the dynamics of these instabilities.

\section{MRI IN THE VGSB}

Following, and extending, the general technique of Latter et al. (2010), we perform a linear stability analysis of the MRI in the framework of the VGSB. In order to derive the equations for the linear mode analysis that lead to the MRI, we assume a homogeneous background magnetic field $\boldsymbol{B}=B_{0} \hat{\boldsymbol{z}}$ and examine the evolution of the perturbations of the form $f(z) \exp (\sigma t)$ for the velocity and magnetic field components $w_{x}, w_{y}, B_{x}, B_{y}$, considering $\delta \rho=w_{z}=0$. Upon substitution in Equations (57)- 60) and linearizing in the perturbation 

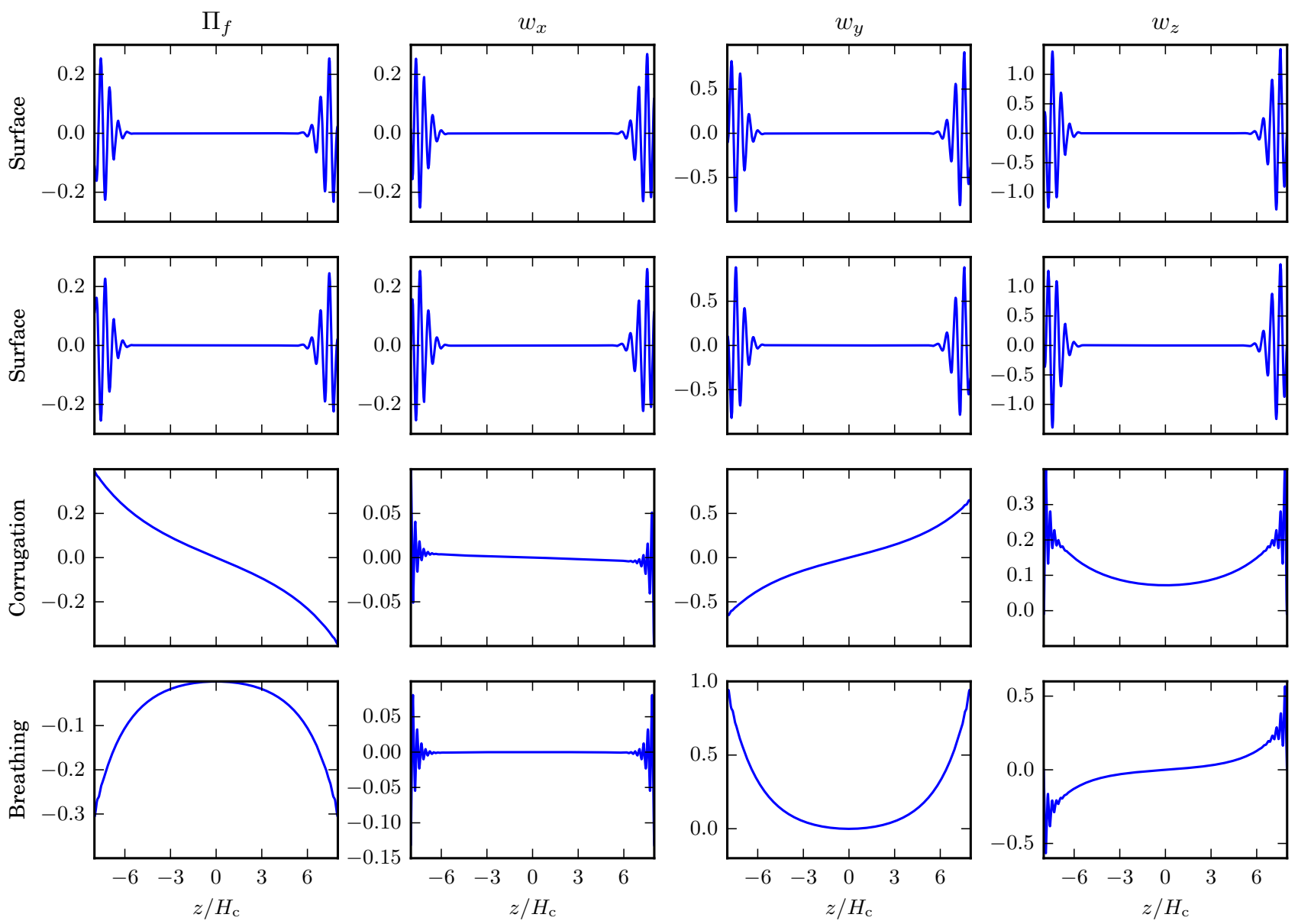

Figure 5. Four example eigenfunctions, transformed to real space, of the VSI, with normalization as given in text. Top Two Rows: the two degenerate fastest growing surface modes with degenerate eigenvalues, both modes have $\sigma \approx 0.184 \pm 0.0316 i$. Third Row: fundamental corrugation mode with $\sigma \approx 0.0265 \pm$ $0.0307 i$. Fourth Row: fundamental breathing mode with $\sigma \approx 0.0376 \pm 0.0433 i$.

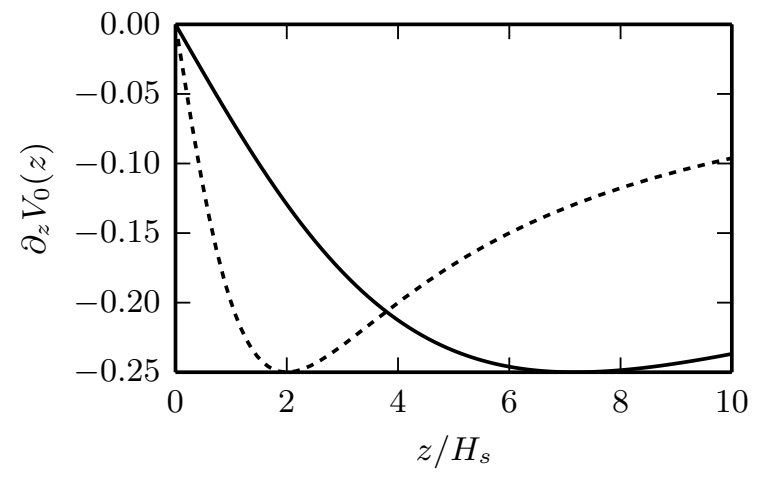

Figure 6. Vertical shear rate in a VGSB model associated with a disk with a global spherical temperature structure, see Section 4.3.2 The solid line corresponds to a thin disk with $\mu+\nu=100$, whereas The dashed line corresponds to a thick disk with $\mu+\nu=10$. amplitudes, the equations for the perturbation are

$$
\begin{aligned}
\sigma w_{x} & =2 \Omega_{0}(z) w_{y}+\frac{B_{0}}{\mu_{0} \rho_{0}(z)} \frac{d B_{x}}{d z}, \\
\sigma w_{y} & =-\left[2 \Omega_{0}(z)+S_{0}(z)\right] w_{x}+\frac{B_{0}}{\mu_{0} \rho_{0}(z)} \frac{d B_{y}}{d z}, \\
\sigma B_{x} & =B_{0} \frac{d w_{x}}{d z} \\
\sigma B_{y} & =S_{0}(z) B_{x}+B_{0} \frac{d w_{y}}{d z} .
\end{aligned}
$$

In a similar way as we did for the VSI, it is convenient to use the scales of length and time provided by $r_{0}$ and $\Omega_{0}(0)^{-1}$ in order to define dimensionless variables, i.e., $\boldsymbol{x} / r_{0} \rightarrow \boldsymbol{x}$ and $t \Omega_{0}(0) \rightarrow t$, etc. Note that the time unit of the growth rate $\sigma$ is $1 / \Omega_{0}(0)$, so its actual value will depend on the parameters of the shearing box model employed.

The set of equations (103)-(106) generalize Equations (12)-(15) in Latter et al. (2010) 9 , to include the ef-

${ }^{9}$ Note a typo in Equation (15) of Latter et al. 2010, where the second term on the left should include a factor of $\Omega$. 
fects of height-dependent angular frequency and shear rate that arise in the context of baroclinic equilibrium disk models. For the sake of simplicity, and in order to make a direct connection with the linear mode analysis in Latter et al. (2010), we have neglected the term proportional to $B_{0} \partial_{z} V_{0}$ on the right hand side of Equation (106), which contributes to the secular evolution of the magnetic field. This is a sensible approximation if the associated modes are localized in height in regions where the vertical shear rate $\partial_{z} V_{0}$ is smaller than the growth rate of the unstable modes. Figure 6 shows the vertical shear rate $\partial_{z} V_{0}$ for two spherical temperature structure VGSB models, and the case which we consider below is roughly compatible with this approximation. However, though for the thin disk case the instability growth is roughly ten times faster than than the vertical shear rate at $H_{s}=1$, this separation is such that the possible effects warrant further exploration in the future.

In order to illustrate how the MRI modes are affected, we examine the spectrum of growing modes present in a disk with spherical temperature structure, $T\left(\sqrt{r^{2}+z^{2}}\right)$, as discussed in Section 4.3.2 taking as an example the $\mu+$ $\nu=100$. These values corresponding to a scale height of $H_{\mathrm{S}} / r_{0}=\sqrt{2} c_{\mathrm{S}}(0) / v_{\mathrm{K} 0}=\sqrt{2}$, and magnetic field strength $B_{0} /\left(r_{0} \Omega_{0}(0) \sqrt{\mu_{0} \rho_{0}(0)}\right)=0.0469$.

We solve the eigenproblem posed by Equations (103)-1106) by discretizing in the infinite domain $-\infty<z<\infty$ in terms of rational Chebyshev cardinal functions on the "roots", or interior grid. For the set of chosen parameters, 200 grid points were sufficient to obtain converged results. We adopted the same set of boundary conditions used in Latter et al. (2010), i.e., $B_{x}=B_{y}$ and $d w_{x} / d z=d w_{y} / d z=0$. To make the problem numerically tractable, we limit $\rho_{0}(z)$ to a minimum value of $10^{-8}$, which is equivalent to limiting the Alfvén speed at high altitudes. The VGSB model of this particular disk model has one more unstable eigenmode than in the SSB. Note that our VGSB analysis yields growth rates scaled by $\Omega_{0}(0)$, which is smaller than the Keplerian frequency. Thus, even though the parameters characterizing the specific VGSB model do not appear explicitly in the linear analysis, the physical values obtained do depend on them. If we consider $\mu=3$, then in the VGSB model analyzed here $\Omega_{0}(0)=\sqrt{\nu /(\nu+\mu)} \Omega_{\mathrm{K} 0}=\sqrt{97 / 100} \Omega_{\mathrm{K} 0}$. This implies that the physical growth rate of the MRI is smaller in the VGSB model of this particular disk than in the SSB. In addition to solving the eigenproblem for the VGSB, we solve the matching problem for the SSB by using an isothermal disk with the same midplane density and scale height $H_{\mathrm{s}}$ as used in the VGSB. The magnetic field and velocity perturbations of the eigenmodes, shown in Figures 7 and 8 , are directionally orthogonal in the SSB, whereas in the VGSB this is not the case. The eigenmodes in the VGSB are generally more complex, and, as it can be seen from these figures, they do not appear to have such a simple ordering in terms of the number of nodes as the SSB eigenmodes do.

\section{DISCUSSION}

The framework of the VGSB allows, for the first time, to develop models for astrophysical disks which are local in radius but are global in height, in that no expansion is made vertically. This is critical to study astrophysical disks where the equilibrium cannot be described by a barotropic equation
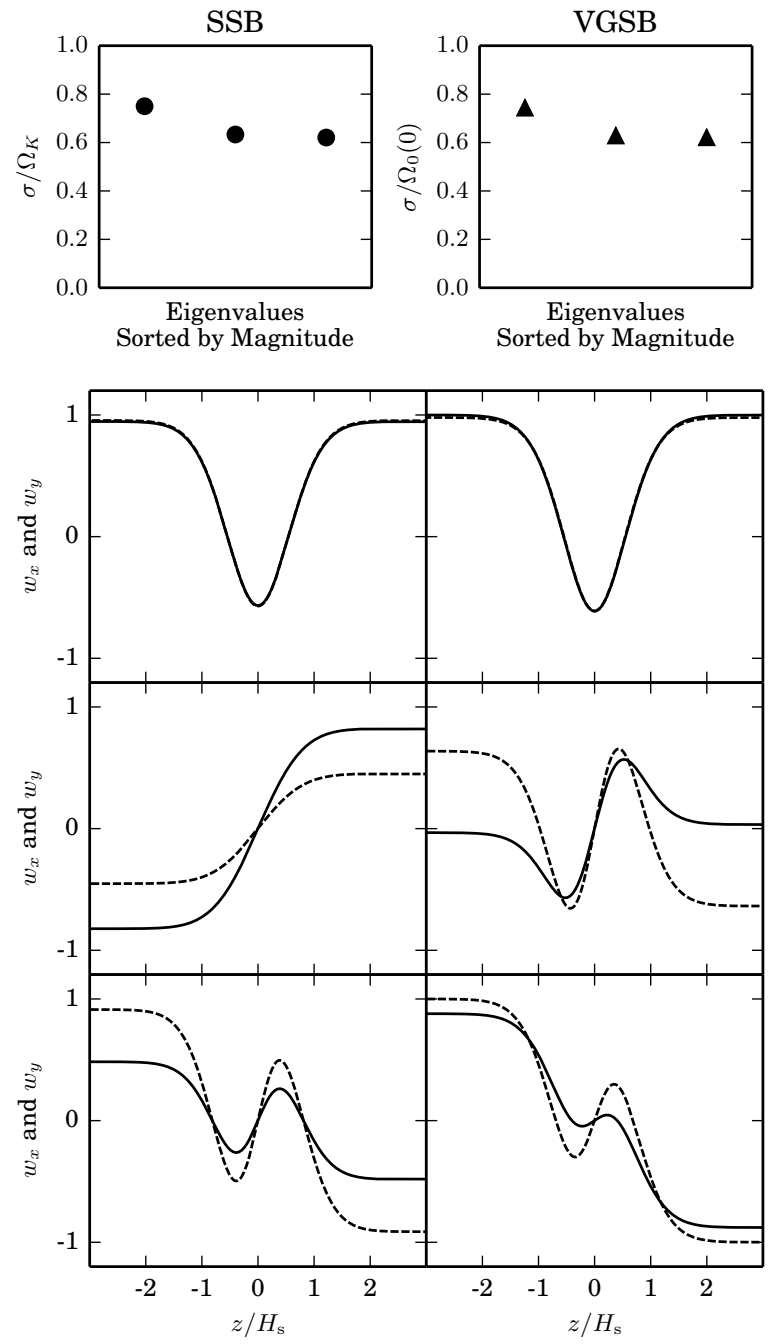

Figure 7. Illustration of the velocity field components $w_{x}$ (solid line) and $w_{y}$ (dashed line) corresponding to the (normalized) MRI modes, which have been ordered from top to bottom from fastest to slowest growth rate, as shown in the uppermost set of panels. The results obtained in the standard shearing box used in Latter et al. (2010) are shown on the left, whereas the modes computed in the VGSB, representing a radially local region of a disk with spherical temperature structure, are shown on the right.

of state, as these, in general, do not rotate on cylinders and are thus not amenable to the SSB framework (see Figure 1). The VGSB naturally accounts for height-dependent radial and vertical flux of fluid and electromagnetic momentum in the azimuthal direction. This is relevant for the dynamics of disks with baroclinic equilibrium structure, especially if these cannot be regarded as thin. Physical domains with a large vertical extent allow for the global magnetic field threading the disk to be efficiently anchored into the surrounding medium. The coupling between stresses and shear, both radial and vertical, can efficiently transport momentum and energy to/from the disk. We envision that these effects will have important consequences, for example, in the study of the coupled dynamics between disks and their coronae and winds.

Because of its inherent local character, the framework provided by the SSB is well suited to study physical processes involving scales that are smaller than the characteristic disk 

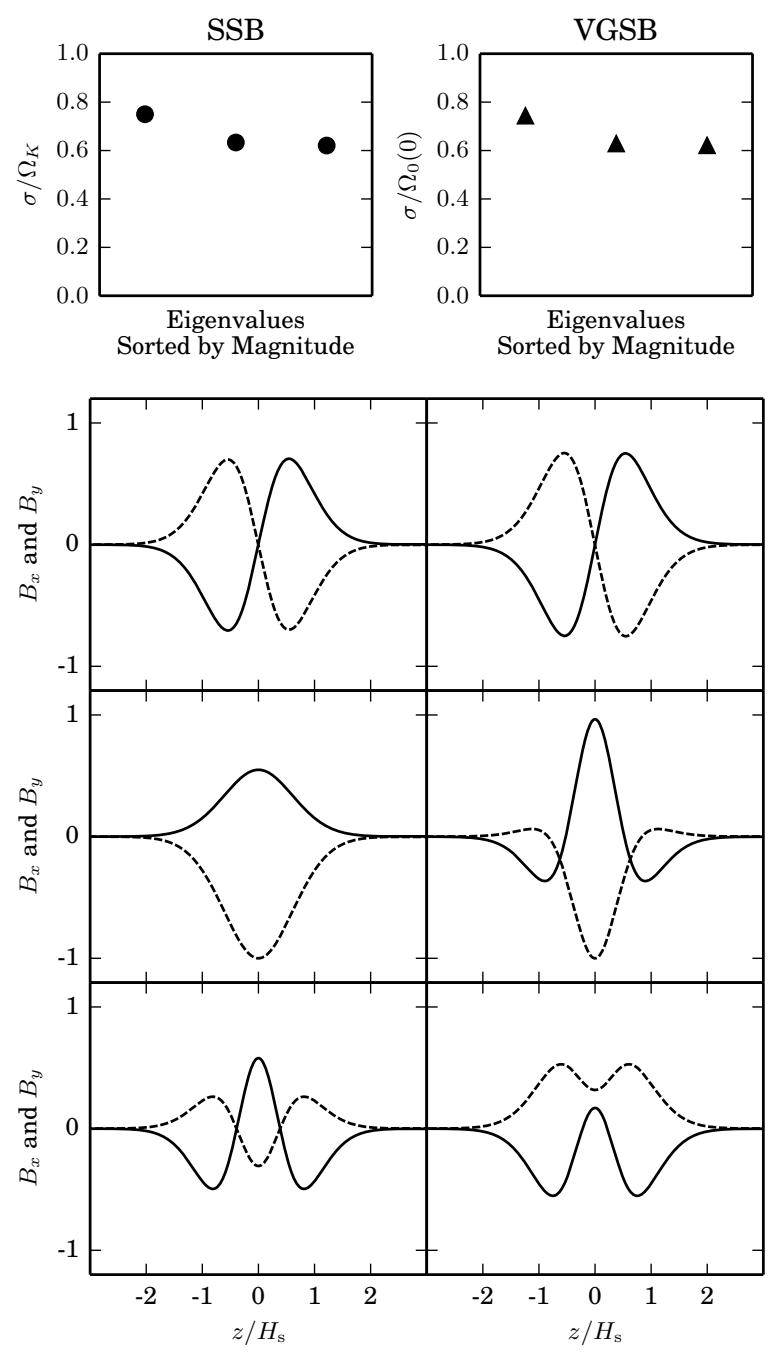

Figure 8. Illustration of the magnetic field components $B_{x}$ (solid line) and $B_{y}$ (dashed line) corresponding to the (normalized) MRI modes. Other details are identical to those described in the caption of Figure 7

scales. Global disk simulations, which are becoming ever more accessible (Flock et al. 2011, Hawley et al. 2011), are useful for understanding the large-scale disk dynamics but face the challenge of resolving the physical processes at small scales. In order to understand how local and global processes interact, it is desirable to devise a framework to bridge local and global approaches. Hence, there have been several initiatives to relax the local character of the SSB.

\subsection{Previous Works Beyond the Standard Shearing Box}

The periodic "shearing disk" annulus introduced in Klahr \& Bodenheimer (2003) relaxes the condition of azimuthal locality by building a radially periodic annulus and remapping quantities across the radial background according to an imposed power-law radial disk structure. This approach involves equations which have explicit coordinate dependence in the direction where the computational domain is shear-periodic. Therefore, the flow properties present a jump at the shearing boundary, which might lead to unphysical effects. In this context, the formulations presented in Brandenburg et al. (1996) and Obergaulinger et al. (2009) complement each other in the sense that they retain the terms accounting for finite curvature but discard global gradients and vice-versa, but both lead to similar issues.

There have been several attempts to make the SSB global in height. Foremost, Goldreich \& Lynden-Bell (1965) treated the case of a polytropic gas, but only made local expansions in the horizontal direction. An early example of modifying the vertical gravity in a SSB is given in Matsuzaki et al. (1997), where the full $z$-dependence of the vertical component of the gravitational force is considered with an isothermal gas. Several other authors have used similar approaches, for both galactic (e.g. Korpi et al. 1999) and Keplerian disks (e.g. Suzuki et al. 2010). These works assume that all the global effects in the vertical direction can be accounted for by modifying only the momentum equation in the $z$-direction and exclusively through the term $\partial_{z} \Phi_{0}(z) \hat{z}$. When the gas in the global equilibrium configuration is strictly baroptropic, it must rotate on cylinders, and the height-independent shear in the SSB is a consistent approximation (given the caveats in Appendix D. Importantly, in the case that the gas is assumed to be isothermal, the SSB provides a consistent approach if the global disk configuration, at all radii, is isothermal. If another equation of state for the hydrostatic structure is used, or if the global configuration of which the shearing box is a small part has a radial temperature gradient, then the VGSB provides a viable framework going beyond these previous approaches, with important modifications beyond the SSB formalism, see Equations (57)-59.

\subsection{Limitations of the VGSB}

The generalization of the SSB to account for the full vertical variation of gravity and the presence of vertical shear, inherent to global baroclinic disk models, does posses limitations. Some of these limitations are also characteristic of the $\mathrm{SSB}$, while others are inherent to the VGSB.

It should be clear that the VGSB, as well as the SSB, framework consists of a set of dynamical equations for the perturbations with respect to a time-independent background representing a smaller section of a steady global disk model. In this approach, by construction, the fluctuations cannot modify the dynamics of the background. This could be considered a serious limitation, however, this kind of approach as embodied in the SSB has proven exceptionally useful as a workhorse to understand a wide variety of phenomena in disks. Another limitation of the VGSB, that is shared by the SSB, is that the local approximation in radius prevents the full consideration curvature terms, which are inherent to the cylindrical geometry usually employed to model disks (see Appendix C).

The set of equations that define the SSB can be obtained by expanding in radius and height either the gravitational potential of a point source or the Keplerian background flow that ensues when radial pressure support can be neglected. In order to derive the equations defining the VGSB we have approximated the background flow itself. The body and frame forces which appear in this formulation relate to a fluid element in that flow with the given global thermodynamics, not the forces on a test particle. Thus, these forces are not the same as an expansion of the tidal potential about a Keplerian orbit. In the special case of a barotropic global equilibrium, the body forces do correspond to a conservative potential, but in general for baroclinic backgrounds this is not the case. The 
advantage of expanding the angular frequency is that its equilibrium profile $\Omega(r, z)$ is sensitive to the equilibrium pressure gradient, enabling access to rotation laws beyond strictly $\mathrm{Ke}$ plerian, in particular those with vertical shear.

The most important limitations of the VGSB stem from the fact that we have opted to explore the consequences of imposing height-dependent shearing-periodic boundary conditions (see Section 3.3). In order to do this, we had to approximate Equation (42) and use Equation (43) instead. Whereas this seems a reasonable approximation, it had in general undesired consequences for some basic conservation laws such as Kelvin's Circulation Theorem and Alfvén's Frozen-in Theorem unless axisymmetry is imposed. In order to consider height-dependent shearing-periodic boundary conditions we also needed to understand under what circumstances it was acceptable to neglect background pressure gradients. Even though the VGSB can be considered to be global in height because there is no need to expand the background in the $z$ direction, the analysis we carried out of the pressure gradients in Appendix $\mathrm{D}$ shows that it is necessary to limit the vertical extent of the domain to $z \lesssim \sqrt{x r_{0}}$. This is more restrictive than $z \lesssim r_{0}$, but nevertheless can accommodate for many scale-heights with the added value of being able to account for the full expression of the vertical gravity and vertical shear within the domain.

The derivation of the VGSB formalism has allowed us to shed light into several subtle issues that are usually not addressed in the context of the SSB, see e.g., Appendices D and E In spite of its limitations, the SSB has proven to be a useful tool to learn about local disk dynamics. We believe that the VGSB will allow the relaxation of some of the assumptions that have been widely adopted by using the SSB. In particular, it will enable the investigation of some dynamical aspects of astrophysical disks that cannot be studied with the SSB and for which full global modeling is too demanding.

\subsection{Applications of the VGSB}

We anticipate that the VGSB framework, summarized in Appendix B, will benefit the modeling of a wide variety of phenomena in astrophysical disks. The advances made possible with respect to the SSB will depend on the nature of the physical phenomena under study, e.g.,:

Hydrodynamic Disk Instabilities - The VGSB framework may be used for the local study of vertical shear instabilities, such as GSF instability (Goldreich \& Schubert 1967, Fricke 1968) and generalizations (Nelson et al. 2013). We have demonstrated in Section 5 that the VGSB captures the correct local linear behavior. It should also be useful to analyze the propagation of hydrodynamic waves in disks (Lubow \& Pringle 1993, Korycansky \& Pringle 1995).

Disk Convection - Vertical convective instabilities in disks (Lin \& Papaloizou 1980) have been studied with the SSB as an angular momentum transport mechanism. Though early studies (Ryu \& Goodman 1992; Cabot 1996; Stone \& Balbus 1996) gave negative results, Lesur \& Ogilvie (2010) have suggested that earlier models are under-resolved, and outward angular momentum transport is possible. The VGSB framework enables to study the effects that vertical shear can have on the long-term evolution of convective motions in a way which is not accessible with the SSB.
Disk Coronae - Previous standard stratified shearing box simulations show the buoyant rise of magnetic field to the upper disk layers (Miller \& Stone 2000; Davis et al. 2010), where it is thought to dissipate giving rise to a hot corona (Galeev et al.1979). If the disk's equilibrium structure is not barotropic, the fact that the shear, i.e., the source of free energy, decreases with height can have important implications for the turbulent disk dynamics and energetics in the disk corona. This could affect the vertical disk structure (Turner 2004; Johansen \& Levin 2008, Blaes et al. 2011) and its corona (Blackman \& Pessah 2009). These effects could be important for thick disks, such as advection dominated accretion flows (ADAFs) (Ichimaru 1977, Narayan et al. 1998. Blandford \& Begelman 1999).

Disk Winds - Standard shearing boxes extended in height have been used to study the MRI (Balbus \& Hawley 1991) as a mechanism for launching disk winds (Suzuki \& Inutsuka 2009; Suzuki et al. 2010, Ogilvie 2012, Moll 2012; Bai \& Stone 2013; Fromang et al. 2013; Lesur et al. 2013) though only in the framework provided by modifying the gravitational forces through the term $\partial_{z} \Phi_{0}(z) \hat{z}$. We have shown in Section 6 that the linear MRI is modified by effects included in the VGSB for disks with baroclinic equilibria. Because the wind dynamics are particularly sensitive to the forces acting on the fluid far away from the midplane, the VGSB provides a more general framework for local studies of this nature.

Interstellar Medium and Galactic Disks - In galactic disks, the VGSB makes it possible to capture critical physics for studying the nonlinear evolution of the magnetorotational, Parker, and magneto-Jeans instabilities (Kim et al. 2002, Piontek \& Ostriker 2005). The framework provided by the VGSB also allows for the inclusion of fundamental height-dependent physical effects that can play an important role in the study of star formation, galactic dynamos, and the structure of the interstellar medium (de Avillez \& Breitschwerdt 2005, Joung \& Mac Low 2006; Gressel et al. 2008).

We are grateful to Tobias Heinemann, whose valuable insights helped us to better understand the subtleties involved in the derivation of the VGSB and to present it in a transparent way. We acknowledge thoughtful comments from Scott Tremaine and Eric Blackman, and discussions with Troels Haugbølle, Åke Nordlund, Andrew Jackson, Oliver Gressel, Orkan M. Umurhan, Gopakumar Mohandas, Henrik Latter, Andrea Mignone, Gianluigi Bodo and Sacha Brun. We are thankful to the anonymous referee, whose tenacious reports led us to better appreciate how the VGSB connects to, and extends, the SSB framework, and to improve the original manuscript significantly. The research leading to these results has received funding from the People Programme (Marie Curie Actions) of the European Union's Seventh Framework Programme (FP7/2007-2013) under REA grant agreement 327995 (CPM), and the European Research Council under the European Union's Seventh Framework Programme (FP/2007-2013) under ERC grant agreement 306614 (MEP). MEP also acknowledges support from the Young Investigator 
Programme of the Villum Foundation.

\section{APPENDIX}

\section{A. DERIVATION OF THE MOMENTUM EQUATION FOR DEPARTURES FROM THE BACKGROUND BULK FLOW}

Here, we provide the algebraic details involved in obtaining the momentum equation (18) starting from Equation (16).

Let us first expand the left-hand side of Equation (16), LHS for short, after substituting $\boldsymbol{v}=V(r, z) \hat{\boldsymbol{\phi}}+\boldsymbol{w}$, we obtain

$$
\begin{aligned}
\text { LHS }= & \left(\frac{\partial}{\partial t}+\boldsymbol{v} \cdot \nabla\right) \boldsymbol{v} \\
= & \frac{\partial \boldsymbol{w}}{\partial t}+(V \hat{\boldsymbol{\phi}}+\boldsymbol{w}) \cdot \nabla(V \hat{\boldsymbol{\phi}}+\boldsymbol{w}) \\
= & \frac{\partial \boldsymbol{w}}{\partial t}+V \hat{\boldsymbol{\phi}} \cdot \nabla(V \hat{\boldsymbol{\phi}})+V \hat{\boldsymbol{\phi}} \cdot \nabla \boldsymbol{w} \\
& +\boldsymbol{w} \cdot \nabla(V \hat{\boldsymbol{\phi}})+\boldsymbol{w} \cdot \nabla \boldsymbol{w} .
\end{aligned}
$$

We now expand the last three terms, recalling that the differential operators involved are in cylindrical coordinates and using the definition of the bulk flow in terms of the angular frequency $V(r, z)=r\left[\Omega(r, z)-\Omega_{\mathrm{F}}\right]$. This yields

$$
\begin{aligned}
\text { LHS }= & \frac{\partial \boldsymbol{w}}{\partial t}-\frac{V^{2}}{r} \hat{\boldsymbol{r}} \\
& +\left[\Omega(r, z)-\Omega_{\mathrm{F}}\right]\left(\frac{\partial w_{r}}{\partial \phi} \hat{\boldsymbol{r}}+\frac{\partial w_{\phi}}{\partial \phi} \hat{\boldsymbol{\phi}}+\frac{\partial w_{z}}{\partial \phi} \hat{\boldsymbol{z}}\right) \\
& -\frac{V w_{\phi}}{r} \hat{\boldsymbol{r}}+\frac{V w_{r}}{r} \hat{\boldsymbol{\phi}} \\
& -\frac{w_{\phi} V}{r} \hat{\boldsymbol{r}}+w_{r} \frac{\partial V}{\partial r} \hat{\boldsymbol{\phi}}+w_{z} \frac{\partial V}{\partial z} \hat{\boldsymbol{\phi}}+\boldsymbol{w} \cdot \nabla \boldsymbol{w}, \\
= & \frac{\partial \boldsymbol{w}}{\partial t}-\Omega^{2}(r, z) r \hat{\boldsymbol{r}}+2 \Omega(r, z) \Omega_{\mathrm{F}} r \hat{\boldsymbol{r}}-\Omega_{\mathrm{F}}^{2} r \hat{\boldsymbol{r}} \\
& +\left[\Omega(r, z)-\Omega_{\mathrm{F}}\right]\left(\frac{\partial w_{r}}{\partial \phi} \hat{\boldsymbol{r}}+\frac{\partial w_{\phi}}{\partial \phi} \hat{\boldsymbol{\phi}}+\frac{\partial w_{z}}{\partial \phi} \hat{\boldsymbol{z}}\right) \\
& -2\left[\Omega(r, z)-\Omega_{\mathrm{F}}\right] w_{\phi} \hat{\boldsymbol{r}}+\left[\Omega(r, z)-\Omega_{\mathrm{F}}\right] w_{r} \hat{\boldsymbol{\phi}} \\
& +w_{r} \frac{\partial r\left[\Omega(r, z)-\Omega_{\mathrm{F}}\right]}{\partial r} \hat{\boldsymbol{\phi}}+w_{z} \frac{\partial V}{\partial z} \hat{\boldsymbol{\phi}}+\boldsymbol{w} \cdot \nabla \boldsymbol{w} \\
= & \frac{\partial \boldsymbol{w}}{\partial t}-\Omega^{2}(r, z) r \hat{\boldsymbol{r}}+2 \Omega(r, z) \Omega_{\mathrm{F}} r \hat{\boldsymbol{r}}-\Omega_{\mathrm{F}}^{2} r \hat{\boldsymbol{r}} \\
& +\left[\Omega(r, z)-\Omega_{\mathrm{F}}\right]\left(\frac{\partial w_{r}}{\partial \phi} \hat{\boldsymbol{r}}+\frac{\partial w_{\phi}}{\partial \phi} \hat{\boldsymbol{\phi}}+\frac{\partial w_{z}}{\partial \phi} \hat{\boldsymbol{z}}\right) \\
& +2 \boldsymbol{\Omega}(r, z) \times \boldsymbol{w}-2 \Omega_{\mathrm{F}} \times \boldsymbol{w}+w_{r} r \frac{\partial \Omega(r, z)}{\partial r} \hat{\boldsymbol{\phi}} \\
& +w_{z} \frac{\partial r \Omega(r, z)}{\partial z} \hat{\boldsymbol{\phi}}+\boldsymbol{w} \cdot \nabla \boldsymbol{w} .
\end{aligned}
$$

This completes the expansion of the left-hand side in terms of the new velocity variable $\boldsymbol{w}$.

We now proceed similarly with the right-hand side of the momentum equation (16), RHS for short, by substituting $\boldsymbol{v}=$
$V(r, z) \hat{\boldsymbol{\phi}}+\boldsymbol{w}$ and expanding

$$
\begin{aligned}
\text { RHS }= & -\Omega^{2}(r, z) r \hat{\boldsymbol{r}}+\frac{1}{\rho_{h}} \frac{\partial P_{h}}{\partial r} \hat{\boldsymbol{r}}+\frac{1}{\rho_{h}} \frac{\partial P_{h}}{\partial z} \hat{\boldsymbol{z}}+\Omega_{\mathrm{F}}^{2} r \hat{\boldsymbol{r}} \\
& -2 \boldsymbol{\Omega}_{\mathrm{F}} \times \boldsymbol{v}-\frac{\nabla P}{\rho}+\frac{1}{\rho} \boldsymbol{J} \times \boldsymbol{B}, \quad(113) \\
= & -\Omega^{2}(r, z) r \hat{\boldsymbol{r}}+\frac{1}{\rho_{h}} \frac{\partial P_{h}}{\partial r} \hat{\boldsymbol{r}}+\frac{1}{\rho_{h}} \frac{\partial P_{h}}{\partial z} \hat{\boldsymbol{z}}+\Omega_{\mathrm{F}}^{2} r \hat{\boldsymbol{r}} \\
& -2 \boldsymbol{\Omega}_{\mathrm{F}} \times(V \hat{\boldsymbol{\phi}}+\boldsymbol{w})-\frac{\nabla P}{\rho}+\frac{1}{\rho} \boldsymbol{J} \times \boldsymbol{B}, \quad(114) \\
= & -\Omega^{2}(r, z) r \hat{\boldsymbol{r}}+\frac{1}{\rho_{h}} \frac{\partial P_{h}}{\partial r} \hat{\boldsymbol{r}}+\frac{1}{\rho_{h}} \frac{\partial P_{h}}{\partial z} \hat{\boldsymbol{z}}-\Omega_{\mathrm{F}}^{2} r \hat{\boldsymbol{r}} \\
& +2 \Omega_{\mathrm{F}} \Omega(r, z) r \hat{\boldsymbol{r}}-2 \boldsymbol{\Omega}_{\mathrm{F}} \times \boldsymbol{w}-\frac{\nabla P}{\rho}+\frac{1}{\rho} \boldsymbol{J} \times \boldsymbol{B} .
\end{aligned}
$$

Equating the left-hand side expression (Equation 112 ) and the right-hand side expression (Equation 115) and canceling out the term $-2 \Omega_{\mathrm{F}} \times \boldsymbol{w}$ which appears on both sides, the momentum Equation (16) becomes, without approximation,

$$
\begin{aligned}
\frac{\partial \boldsymbol{w}}{\partial t} & +\left[\Omega(r, z)-\Omega_{\mathrm{F}}\right]\left(\frac{\partial w_{r}}{\partial \phi} \hat{\boldsymbol{r}}+\frac{\partial w_{\phi}}{\partial \phi} \hat{\boldsymbol{\phi}}+\frac{\partial w_{z}}{\partial \phi} \hat{\boldsymbol{z}}\right) \\
& +\boldsymbol{w} \cdot \nabla \boldsymbol{w}+w_{r} r \frac{\partial \Omega(r, z)}{\partial r} \hat{\boldsymbol{\phi}} \\
& +w_{z} \frac{\partial r \Omega(r, z)}{\partial z} \hat{\boldsymbol{\phi}}+2 \boldsymbol{\Omega}(r, z) \times \boldsymbol{w} \\
& =\frac{\nabla P_{h}}{\rho_{h}}-\frac{\nabla P}{\rho}+\frac{1}{\rho} \boldsymbol{J} \times \boldsymbol{B},
\end{aligned}
$$

which corresponds to Equation (18).

As stated in Section 3.1.1 a cancellation of terms from the left and right-hand sides of the momentum equation results in the term $\boldsymbol{\Omega}(r, z) \times \boldsymbol{w}$ that looks similar to the Coriolis acceleration, i.e., $\boldsymbol{\Omega}_{\mathrm{F}} \times \boldsymbol{w}$, but with the angular frequency $\boldsymbol{\Omega}(r, z)$ taking the place of the constant angular frequency of the rotating frame $\Omega_{\mathrm{F}}$.

\section{B. SUMMARY OF THE VGSB EQUATIONS}

Here we provide a self-contained summary of the equations defining the VGSB.

VGSB Equations - The continuity, momentum, induction, and energy equations in the VGSB are, respectively, given by

$$
\begin{gathered}
\mathcal{D}_{0} \rho+\nabla \cdot(\rho \boldsymbol{w})=0, \\
\left(\mathcal{D}_{0}+\boldsymbol{w} \cdot \nabla\right) \boldsymbol{w}+w_{z} \frac{\partial V_{0}(z)}{\partial z} \hat{\boldsymbol{y}}=-2 \Omega_{0}(z) \hat{\boldsymbol{z}} \times \boldsymbol{w} \\
-S_{0}(z) w_{x} \hat{\boldsymbol{y}}-\frac{\nabla P}{\rho}-\frac{\partial \Phi_{0}(z)}{\partial z} \hat{\boldsymbol{z}}+\frac{1}{\rho} \boldsymbol{J} \times \boldsymbol{B}, \\
\left(\mathcal{D}_{0}+\boldsymbol{w} \cdot \nabla\right) \boldsymbol{B}-B_{z} \frac{\partial V_{0}(z)}{\partial z} \hat{\boldsymbol{y}}=S_{0}(z) B_{x} \hat{\boldsymbol{y}} \\
+(\boldsymbol{B} \cdot \nabla) \boldsymbol{w}-\boldsymbol{B}(\nabla \cdot \boldsymbol{w}), \\
\mathcal{D}_{0} e+\nabla \cdot(e \boldsymbol{w})=-P(\nabla \cdot \boldsymbol{w}) .
\end{gathered}
$$

Here, $\rho$ is the mass density, $\boldsymbol{w} \equiv \boldsymbol{v}-\left[V_{0}(z)+S_{0}(z) x\right] \hat{\boldsymbol{y}}$ is the velocity with respect to the local bulk flow in the disk, $B$ is the magnetic field, and $e$ is the internal energy density. The 
pressure $P$ is determined as a function of $\rho$ and $e$, through an appropriate equation of state. The current density is $\boldsymbol{J} \equiv$ $\nabla \times \boldsymbol{B} / \mu_{0}$, with $\mu_{0}$ a constant dependent on the unit system adopted. All the operators in the VGSB equations are defined in a cartesian coordinate system centered at the fiducial radius $r_{0}$.

For barotropic background equilibrium disc structures, the above equations can be used in fully three dimensions, while for baroclinic background equilibrium disc structures, these equations must by restricted to axisymmetric solutions ( $y$ invariant).

VGSB Definitions - The equations defining the VGSB framework depend on a number of functions of the coordinate $z$, which result from local radial expansions of the global disk model around the radius $r_{0}$. The height-dependent advection by the background shear, $\mathcal{D}_{0}(z)$, is given by

$$
\mathcal{D}_{0}(z) \equiv \frac{\partial}{\partial t}+\left[V_{0}(z)+S_{0}(z) x\right] \frac{\partial}{\partial y},
$$

where $\Omega_{0}(z), V_{0}(z)$, and $S_{0}(z)$ correspond, respectively, to the local values of the background azimuthal velocity, angular frequency, and background shear flow, which are all derived from the angular frequency of the global disk model $\Omega(r, z)$, i.e.,

$$
\begin{aligned}
V_{0}(z) & \equiv r_{0}\left[\Omega_{0}(z)-\Omega_{0}(0)\right], \\
\Omega_{0}(z) & \equiv \Omega\left(r_{0}, z\right), \\
S_{0}(z) & \left.\equiv r_{0} \frac{\partial \Omega(r, z)}{\partial r}\right|_{r=r_{0}} .
\end{aligned}
$$

The gravitational potential in the $\operatorname{VGSB} \Phi_{0}(z)$ is given by the value of the global gravitational potential $\Phi(r, z)$ at $r_{0}$

$$
\Phi_{0}(z) \equiv \Phi\left(r_{0}, z\right) \text {. }
$$

VGSB Boundary Conditions - For a domain with size $L_{x} \times$ $L_{y} \times L_{z}$ the horizontal boundary conditions are

$$
\begin{aligned}
& f(x, y, z, t)=f\left(x+L_{x}, y+S_{0}(z) L_{x} t, z, t\right), \\
& f(x, y, z, t)=f\left(x, y+L_{y}, z, t\right) .
\end{aligned}
$$

which correspond to a generalization of the shearing-periodic boundary conditions adopted in the SSB.

\section{NEGLECTING CURVATURE TERMS}

The cylindrical coordinate system that we adopt to describe the global disk model leads naturally to the presence of the quadratic terms $-w_{y}^{2} / r_{0} \hat{\boldsymbol{x}}, w_{x} w_{y} / r_{0} \hat{\boldsymbol{y}},-B_{y}^{2} / r_{0} \hat{\boldsymbol{x}}$, and $B_{x} B_{y} / r_{0} \hat{\boldsymbol{y}}$ in the momentum equation (31). All of these terms are usually neglected in the SSB. The terms related to the velocity field can be neglected safely when they are small compared to the corresponding components of the acceleration $-w_{y} \Omega_{0}(z) \hat{\boldsymbol{x}}$ and $w_{x} \Omega_{0}(z) \hat{\boldsymbol{y}}$. Both of these conditions lead to the inequality $w_{y} \ll r_{0} \Omega_{0}(z)$. The magnetic terms $-B_{y}^{2} / r_{0} \hat{\boldsymbol{x}}$ and $+B_{x} B_{y} / r_{0} \hat{\boldsymbol{y}}$ in Equation 31 are usually absent in local studies (see Brandenburg et al.1996 for an exception). If the magnetic field is sufficiently sub-thermal everywhere in the domain, these terms can be neglected (Pessah \& Psaltis 2005). However, they have been commonly neglected even when this is not the case. This is perhaps because it is not obvious that retaining them will lead to a physically consistent problem in the framework of the shearing box. Consider, for example, the case of a shearing box in which a strong net azimuthal field develops as a consequence of the local disk dynamics. This could lead to the force arising from the term $-B_{y}^{2} / r_{0} \hat{\boldsymbol{x}}$ to increase with time. However, this force cannot be balanced by an increase in the centripetal acceleration as the latter is fixed by the choice of the bulk flow (e.g. Lubow \& Spruit 1995). Note that all of this statements regarding the quadratic terms that reminisce the curvilinear nature of the original cylindrical coordinate system are independent of whether the SSB, or the vertically global version developed in this paper, is considered.

\section{NONDIMENSIONALIZATION AND ORDERING OF THE HYDRODYNAMIC MOMENTUM EQUATION}

A more rigorous description of the approximations that lead to the equations for the VGSB can be made by introducing nondimensional variables in order to expose the hierarchy of the various terms involved. Here, we only deal with the hydrodynamic part of the momentum equation, for the magnetic components we make the assumption that the magnetic field are weak enough to not effect the global equilibrium background configuration. Our approach follows and generalizes Umurhan \& Regev (2004).

The hydrodynamic part of the momentum equation 22 is given by

$$
\begin{gathered}
(\mathcal{D}+\boldsymbol{w} \cdot \nabla) \boldsymbol{w}=-2 \Omega(r, z) \hat{\boldsymbol{z}} \times \boldsymbol{w}-S(r, z) w_{r} \hat{\boldsymbol{\phi}} \\
-w_{z} \frac{\partial V(r, z)}{\partial z} \hat{\boldsymbol{\phi}}+\frac{\nabla P\left(\rho_{h}, e_{h}\right)}{\rho_{h}}-\frac{\nabla P(\rho, e)}{\rho}
\end{gathered}
$$

We define a horizontal length scale $\lambda_{r}$, a vertical length scale $\lambda_{z}$, time scale $\Omega_{0}^{-1}$, pressure scale $P_{0}$, and density scale $\rho_{0}$. Horizontal velocities are nondimensionalized by $\lambda_{r} \Omega_{0}$ and vertical velocities by $\lambda_{z} \Omega_{0}$. We use primes to denote normalized quantities, like $w_{r}^{\prime}$ the radial component of the fluctuation velocity, and $\partial_{r^{\prime}}$ the radial partial derivative with respect to the radial nondimensional length, with radial length nondimensionalized as $x^{\prime}=x / \lambda_{r}$. The components of the momentum equation are thus

$$
\begin{aligned}
\left(\mathcal{D}^{\prime}+\right. & \left.\boldsymbol{w}^{\prime} \cdot \nabla\right) w_{r}^{\prime}=2 \Omega^{\prime}(r, z) w_{\phi}^{\prime} \\
& +\left(\frac{\epsilon}{\delta_{x}}\right)^{2}\left[\frac{\partial_{r^{\prime}} P^{\prime}\left(\rho_{h}, e_{h}\right)}{\rho_{h}^{\prime}}-\frac{\partial_{r^{\prime}} P^{\prime}(\rho, e)}{\rho^{\prime}}\right], \\
\left(\mathcal{D}^{\prime}+\boldsymbol{w}^{\prime} \cdot \nabla\right) w_{\phi}^{\prime}=-2 \Omega^{\prime}(r, z) w_{r}^{\prime}-S^{\prime}(r, z) w_{r}^{\prime} & \rho^{\prime} \\
& +\left(\frac{\epsilon}{\delta_{x}}\right)^{2}\left[-\frac{\left(1 / r^{\prime}\right) \partial_{\phi} P^{\prime}(\rho, e)}{\left(\mathcal{D}^{\prime}+\boldsymbol{w}^{\prime} \cdot \nabla\right) w_{z}^{\prime}=}\right. \\
& \left(\frac{\epsilon}{\delta_{z}}\right)^{2}\left[\frac{\partial_{z^{\prime}} P^{\prime}\left(\rho_{h}, e_{h}\right)}{\rho_{h}^{\prime}}-\frac{\partial_{z^{\prime}} P^{\prime}(\rho, e)}{\rho^{\prime}}\right] .
\end{aligned}
$$


where we have introduced the dimensionless parameters

$$
\begin{aligned}
\epsilon & \equiv \frac{1}{\Omega_{0} r_{0}} \sqrt{\frac{P_{0}}{\rho_{0}}}, \\
\delta_{x} & \equiv \frac{\lambda_{r}}{r_{0}}, \\
\delta_{z} & \equiv \frac{\lambda_{z}}{r_{0}} .
\end{aligned}
$$

Note that $\Omega_{0}$ is most readily identified as the $\Omega(r, z)$ appearing at the location of the dynamics in question, not the Keplerian frequency. Thus $\epsilon$ is not directly the usual nondimensional scale height. Taylor expanding in small $\delta_{x}$ about $r_{0}$, keeping only lowest order terms, and transforming to cartesian coordinates as this allows (Section 3.2 ) yields

$$
\begin{aligned}
\left(\mathcal{D}_{0}^{\prime}\right. & \left.+\boldsymbol{w}^{\prime} \cdot \nabla\right) w_{x}^{\prime}=2 \Omega_{0}^{\prime}(z) w_{y}^{\prime} \\
& +\left(\frac{\epsilon}{\delta_{x}}\right)^{2}\left[\frac{\partial_{x^{\prime}} P^{\prime}\left(\rho_{h}, e_{h}\right)}{\rho_{h}^{\prime}}-\frac{\partial_{x^{\prime}} P^{\prime}(\rho, e)}{\rho^{\prime}}\right], \\
\left(\mathcal{D}_{0}^{\prime}\right. & \left.+\boldsymbol{w}^{\prime} \cdot \nabla\right) w_{y}^{\prime}=-2 \Omega_{0}^{\prime}(z) w_{x}^{\prime}-S_{0}^{\prime}(z) w_{x}^{\prime} \\
& +\left(\frac{\epsilon}{\delta_{x}}\right)^{2}\left[-\frac{\partial_{y^{\prime}} P^{\prime}(\rho, e)}{\rho^{\prime}}\right], \\
\left(\mathcal{D}_{0}^{\prime}\right. & \left.+\boldsymbol{w}^{\prime} \cdot \nabla\right) w_{z}^{\prime}= \\
& \left(\frac{\epsilon}{\delta_{z}}\right)^{2}\left[\frac{\partial_{z^{\prime}} P^{\prime}\left(\rho_{h}, e_{h}\right)}{\rho_{h}^{\prime}}-\frac{\partial_{z^{\prime}} P^{\prime}(\rho, e)}{\rho^{\prime}}\right] .
\end{aligned}
$$

We now decompose the pressure and density into their hydrostatic part and a their associated fluctuation as

$$
\begin{aligned}
P^{\prime} & \equiv P_{1}^{\prime}+P_{h}^{\prime}, \\
\rho^{\prime} & \equiv \rho_{1}^{\prime}+\rho_{h}^{\prime} .
\end{aligned}
$$

The components of the momentum equation then become

$$
\begin{aligned}
& \left(\mathcal{D}_{0}^{\prime}+\boldsymbol{w}^{\prime} \cdot \nabla\right) w_{x}^{\prime}=2 \Omega_{0}^{\prime}(z) w_{y}^{\prime} \\
& \quad+\left(\frac{\epsilon}{\delta_{x}}\right)^{2}\left[\frac{\rho_{1}^{\prime} \partial_{x^{\prime}} P_{h}^{\prime}}{\rho_{h}^{\prime}\left(\rho_{h}^{\prime}+\rho_{1}^{\prime}\right)}-\frac{\partial_{x^{\prime}} P_{1}^{\prime}}{\rho_{h}^{\prime}+\rho_{1}^{\prime}}\right], \\
& \left(\mathcal{D}_{0}^{\prime}+\boldsymbol{w}^{\prime} \cdot \nabla\right) w_{y}^{\prime}=-2 \Omega_{0}^{\prime}(z) w_{x}^{\prime}-S_{0}^{\prime}(z) w_{x}^{\prime} \\
& \quad+\left(\frac{\epsilon}{\delta_{x}}\right)^{2}\left[-\frac{\partial_{y^{\prime}} P_{1}^{\prime}}{\rho_{h}^{\prime}+\rho_{1}^{\prime}}\right], \\
& \left(\mathcal{D}_{0}^{\prime}+\boldsymbol{w}^{\prime} \cdot \nabla\right) w_{z}^{\prime}= \\
& \quad\left(\frac{\epsilon}{\delta_{z}}\right)^{2}\left[\frac{\rho_{1}^{\prime} \partial_{z^{\prime}} P_{h}^{\prime}}{\rho_{h}^{\prime}\left(\rho_{h}^{\prime}+\rho_{1}^{\prime}\right)}-\frac{\partial_{z^{\prime}} P_{1}^{\prime}}{\rho_{h}^{\prime}+\rho_{1}^{\prime}}\right] .
\end{aligned}
$$

The fluctuation pressure terms have the same scaling as the background hydrostatic pressure terms, as in Umurhan \& Regev (2004). Using the expression for hydrostatic equilibrium given by Equation (14, we obtain the form of the radial and vertical components of the acceleration as

$$
\begin{aligned}
& \left(\frac{\epsilon}{\delta_{x}}\right)^{2} \frac{1}{\rho_{h}^{\prime}} \frac{\partial P_{h}^{\prime}}{\partial x^{\prime}}= \\
& \quad-\frac{\left(1+\delta_{x} x^{\prime}\right)}{\delta_{x}}\left[\Omega^{\prime 2}-\Omega_{\mathrm{K} 0}^{\prime 2}\left[\left(1+\delta_{x} x^{\prime}\right)^{2}+\delta_{z}^{2} z^{\prime 2}\right]^{-3 / 2}\right],
\end{aligned}
$$

$$
\left(\frac{\epsilon}{\delta_{z}}\right)^{2} \frac{1}{\rho_{h}^{\prime}} \frac{\partial P_{h}^{\prime}}{\partial z^{\prime}}=-\Omega_{\mathrm{K} 0}^{\prime 2} z^{\prime}\left[\left(1+\delta_{x} x^{\prime}\right)^{2}+\left(\delta_{z} z^{\prime}\right)^{2}\right]^{-3 / 2} .
$$

The vertical acceleration depends only on the gravitational potential, but the radial acceleration depends on the thermodynamics of the hydrostatic equilibrium though the nondimensionalized rotation $\Omega^{\prime}$.

In order to illustrate the procedure for determining the appropriate expansion of the radial acceleration, we work with the structure of the hydrostatic background provided by the cylindrical temperature profile $T(r)$, as in Section 4.3.1 This thermal structure contains a barotropic equilibrium as a special case. The nondimensional version of the rotation law given by Equation 80 is

$$
\begin{aligned}
\Omega^{\prime 2}= & \Omega_{\mathrm{K} 0}^{\prime 2}\left(1+\delta_{x} x^{\prime}\right)^{-3}\left(+(p+q) \epsilon^{2}\left(1+\delta_{x} x^{\prime}\right)^{q+1}\right. \\
& \left.+q\left[1-\frac{\left(1+\delta_{x} x^{\prime}\right)}{\sqrt{\left(1+\delta_{x} x^{\prime}\right)^{2}+\delta_{z}^{2} z^{\prime 2}}}\right]\right)
\end{aligned}
$$

When $q=0$ this structure is isothermal, and barotropic, and when $\epsilon \ll 1$ the disk is thin, with the rotation close to Keplerian.

\section{D.1. Case of SSB and VGSB for a Globally Isothermal Background}

In order to connect our analysis with Umurhan \& Regev (2004), who considered the case with $\epsilon=0$ and $\delta_{x}=\delta_{z}$, we examine explicitly here the globally isothermal case with $q=$ 0 . Even though the equations that we presented for the VGSB do not explicitly involve expansions in the vertical direction, at this point in the analysis we do make such an expansion in order to assess the conditions under which it is acceptable to neglect the radial pressure gradients that need to be discarded in order to impose shearing-periodic boundary conditions, as discussed in Section 3.3. Thus, the results of this section apply equally to the VGSB model for a globally isothermal (barotropic) disk, as well as to the SSB.

Expanding the radial and vertical components of the acceleration about $\delta_{x}=0$ and $\delta_{z}=0$ we obtain, to leading order,

$$
\begin{aligned}
\Omega_{\mathrm{K} 0}^{\prime-2}\left(\frac{\epsilon}{\delta_{x}}\right)^{2} \frac{1}{\rho_{h}^{\prime}} \frac{\partial P_{h}^{\prime}}{\partial x^{\prime}} & \simeq-\frac{p \epsilon^{2}}{\delta_{x}}+p \epsilon^{2} x^{\prime}-p \epsilon^{2} x^{\prime 2} \delta_{x} \\
+p \epsilon^{2} x^{\prime 3} \delta_{x}^{2} & -\frac{3 z^{\prime 2}}{2 \delta_{x}} \delta_{z}^{2}+6 x^{\prime} z^{\prime 2} \delta_{z}^{2}+\ldots, \quad \\
\Omega_{\mathrm{K} 0}^{\prime-2}\left(\frac{\epsilon}{\delta_{z}}\right)^{2} \frac{1}{\rho_{h}^{\prime}} \frac{\partial P_{h}^{\prime}}{\partial z^{\prime}} & \simeq-z^{\prime}+3 x^{\prime} z^{\prime} \delta_{x}+\frac{3}{2} z^{\prime 3} \delta_{z}^{2}+\ldots
\end{aligned}
$$

It is evident, as any finite domain contains arbitrarily small $\delta_{x}$, that the only rigorous limit in which we can strictly justify discarding every term on the right hand side of equation (146) is the one corresponding to a disk with a constant midplane density, $p=0$, and/or infinitely thin, $\epsilon=0$, in which the characteristic scales of interest in the vertical direction vanish identically, $\delta_{z}=0$. This is certainly not an interesting limit, especially in the case where $\delta_{x}=\delta_{z}$. Perhaps this is the reason for which these terms are usually discarded in the standard 
shearing box formalism, even though they cannot be strictly neglected in a disk with a radial density gradient and/or nonvanishing scale height when the vertical extent of the domain does not vanish identically.

Having this caveat in mind, let us examine under what conditions we can argue that the terms proportional to $\delta_{x}$ and $\delta_{z}$ are sufficiently small that it is acceptable to neglect them. This requires understanding when each of the dimensionless terms in question is small compared to unity. In equation $(146)$ for the radial acceleration, the first term can be argued to be sufficiently small for $\delta_{x} \ll 1$ provided that $p \epsilon^{2} / \delta_{x} \ll 1$, which implies $p \ll \rho_{0}\left(r_{0}^{2} \Omega_{0}^{2}\right) / P_{0} \sim\left(r_{0} \Omega_{0} / c_{\mathrm{S}}\right)^{2}$. This condition can be satisfied for power-law indices $p$ of order unity. The second term, of order $\delta_{x}^{0}$, is small based on the same condition as $x^{\prime}$ is at most of order unity. The third and fourth terms are clearly small for $\delta_{x} \ll 1$. The fifth term in this equation is more difficult to deal with because it scales with the ratio $\delta_{z}^{2} / \delta_{x}$. In the case considered by Umurhan \& Regev (2004), because $\delta_{x}=\delta_{z}$, this term is simply proportional to $\delta_{x}$, and it can be directly neglected in the limit $\delta_{x} \ll 1$, along with the higher order terms in the radial acceleration. When the assumption $\delta_{x}=\delta_{z}$ is relaxed, the second term in the radial acceleration is small when $z^{\prime 2} \delta_{z}^{2} \ll \delta_{x}$. Restoring dimensional quantities, this condition becomes $z \ll \sqrt{\lambda_{x} r_{0}}<\sqrt{x r_{0}}$. This implies that the fifth term in the equation for the radial acceleration is small provided that the vertical extent of the domain is smaller than the geometric mean between its radial extent and the radial location of the box. Note that this condition is more restrictive that requesting that $z<r_{0}$, but far less restrictive than imposing that $z$ vanishes identically. The higher order terms, such as the sixth term on the right-hand side of equation (146), are negligible provided that $x \ll r_{0}$. The vertical acceleration in equation (147) does not depend on the parameters $p$ or $\epsilon$. In this case, it is straightforward to neglect higher order terms proportional to $\delta_{x}$ and $\delta_{z}$ with respect to the leading term $z^{\prime}$.

\section{D.2. Case of VGSB for Cylindrical Temperature Profiles}

Here, we generalize the above result in order to include a baroclinic background with non-zero radial temperature power law index $q$. The generalization of Equation (146) is

$$
\begin{aligned}
\Omega_{\mathrm{K} 0}^{\prime-2} & \left(\frac{\epsilon}{\delta_{x}}\right)^{2} \frac{1}{\rho_{h}^{\prime}} \frac{\partial P_{h}^{\prime}}{\partial x^{\prime}} \simeq-\frac{(p+q) \epsilon^{2}}{\delta_{x}}+(1-q)(p+q) \epsilon^{2} x^{\prime} \\
& -\frac{1}{2}(q-2)(q-1)(p+q) \epsilon^{2} x^{\prime 2} \delta_{x} \\
& -\frac{1}{6}(q-3)(q-2)(q-1)(p+q) \epsilon^{2} x^{\prime 3} \delta_{x}^{2} \\
& -(3+q) \frac{z^{\prime 2} \delta_{z}^{2}}{2 \delta_{x}}+(6+2 q) x^{\prime} z^{\prime 2} \delta_{z}^{2}+\ldots
\end{aligned}
$$

It is clear that there is a one-to-one correspondence between each of the terms present in Equation (148) and the ones appearing in Equation (146). For any reasonable value of $q$, the numerical coefficients in both equations are of the same order. Because of this, the conditions required to neglect each of the terms in Equation (148) and Equation (146) are, within factors of order unity, identical. The equation for the expansion of the vertical pressure gradient is independent of $q$ and thus identical to Equation (147).
From the analysis in this appendix, we thus conclude that, within factors of order unity, the requirements to neglect the radial background pressure gradients in the VGSB are expected to be similar to the ones involved in the SSB.

\section{E. POTENTIAL VORTICITY AND ERTEL'S THEOREM}

In previous sections of this paper, we have addressed the impact that the approximations embodied in the VGSB framework have on Kelvin's Circulation Theorem and Alfvén's Frozen-in Theorem and showed that these are satisfied if the fluid is barotropic or axisymmetric. Another important conservation law is given by Ertel's Theorem, which governs the evolution of the potential vorticity $\left(\boldsymbol{\omega}_{a} \cdot \nabla A\right) / \rho$, where $\boldsymbol{\omega}_{a}=\nabla \times \boldsymbol{v}+2 \Omega_{\mathrm{F}}$ is the absolute vorticity and $A$ is a fluid property advected with the flow, according to (Pedlosky 1982)

$$
\left(\frac{\partial}{\partial t}+\boldsymbol{v} \cdot \nabla\right)\left[\frac{\boldsymbol{\omega}_{a}}{\rho} \cdot \nabla A\right]=\frac{\nabla \rho \times \nabla P}{\rho^{3}} \cdot \nabla A .
$$

Note that for any conserved scalar field $A$, Ertel's Theorem leads to a conservation law for the potential vorticity if the flow is barotropic, i.e., $\nabla \rho \times \nabla P=0$. If $A$ is taken to be the specific entropy $s$ in an isentropic flow, i.e. $(\partial / \partial t+\boldsymbol{v}$. $\nabla) s=0$, Ertel's Theorem provides a conservation law for the potential vorticity even if the flow is baroclinic, because $(\nabla \rho \times \nabla P) \cdot \nabla s=0$.

In order to shed light into the implications that the approximations embodied in the VGSB have for Ertel's Theorem let us examine the evolution equation for a PV-type quantity defined, in terms of the VGSB background velocity field $\boldsymbol{V}(x, z)=\left[V_{0}(z)+x S_{0}(z)\right] \hat{\boldsymbol{y}}$ and the velocity fluctuations $\boldsymbol{w}$, as

$$
\begin{aligned}
& \frac{\boldsymbol{\omega}_{a}}{\rho} \cdot \nabla A= \\
& \quad \frac{1}{\rho}\left\{\nabla \times\left[V_{0}(z) \hat{\boldsymbol{y}}+S_{0}(z) x \hat{\boldsymbol{y}}+\boldsymbol{w}\right]+2 \boldsymbol{\Omega}_{\mathrm{F}}\right\} \cdot \nabla A .
\end{aligned}
$$

We can assess when such a quantity obeys a conservation law in the form of Equation (149). Starting from the approximate VGSB momentum equation (58), transforming from the fluctuation velocity $\boldsymbol{w}$ to the velocity $\boldsymbol{v}=\left[V_{0}(z)+x S_{0}(z)\right] \hat{\boldsymbol{y}}+\boldsymbol{w}$, and following the usual steps for deriving a potential vorticity evolution equation (Pedlosky 1982), we arrive to the following evolution equation

$$
\begin{aligned}
& \left(\frac{\partial}{\partial t}+\boldsymbol{v} \cdot \nabla\right)\left[\frac{\boldsymbol{\omega}_{a}}{\rho} \cdot \nabla A\right]= \\
& \quad\left[\frac{1}{\rho} \nabla \times\left\{2 \Omega_{\mathrm{F}} \hat{\boldsymbol{z}} \times\left[V_{0}(z)+S_{0}(z) x\right] \hat{\boldsymbol{y}}\right.\right. \\
& \left.\left.\quad+2\left(\Omega_{\mathrm{F}}-\Omega_{0}(z)\right) \hat{\boldsymbol{z}} \times \boldsymbol{w}+w_{z} \frac{\partial S_{0}(z)}{\partial z} x \hat{\boldsymbol{y}}\right\}\right] \cdot \nabla A \\
& \quad+\frac{\nabla \rho \times \nabla P}{\rho^{3}} \cdot \nabla A .
\end{aligned}
$$

The first term on the right-hand side can be non-zero, so this is not in general a conservation law of the form Equation (149). This term has three components within it, the first being a non- 
conservative tidal force, the second being a coriolis-like force which remains from the velocity transformation, and the final term being due to the approximation made in Equation (43). A coriolis-like force remains from the velocity transform from $\boldsymbol{w}$ to $\boldsymbol{v}$ because in Cartesian coordinates it is just a linear velocity boost in $\hat{\boldsymbol{y}}$, and the VGSB momentum equation contains a coriolis-like term which is proportional to the $\hat{\boldsymbol{y}}$ velocity. Interestingly, when a velocity transformation is done in cylindrical coordinates with an analogous change in the $\hat{\phi}$ velocity, the matching change in the coriolis term cancels with components of the cylindrical coordinate advection operator on the left-hand side, as was seen in Appendix A In the case of a VGSB model with a height-independent $\Omega_{0}(z)$ or an SSB, the first term on the right-hand side of Equation (151) is zero, as the first two components are conservative and curl-free, and the third component is zero. In those cases, this applies for any choice of $\Omega_{\mathrm{F}}$, as $\Omega_{0}$ is constant. Thus in those cases this PV-type quantity is conserved in the same sense as in Equation (149). Beyond simply not being in general in the form of a conservation law, Equation (151) also has the property that the Lagrangian derivative term with $\partial / \partial t+\boldsymbol{v} \cdot \nabla$ is not zero when the velocity fluctuations $\boldsymbol{w}$ are zero. This is because the quantity defined in Equation (150) contains both the background velocity and the fluctuation velocity. Note that in the absence of fluctuations in an axisymmetric flow, i.e., $\partial_{y} \equiv 0$, and the first term on the right-hand side of Equation (151) does vanish.

Understanding the implications of the source terms that would appear in general on the right-hand side of Equation (151) is beyond the scope of this work. In the reminder of this section we have a more modest goal which consists of assessing how the approximations leading to the VGSB impact the dynamics of a PV-type quantity $(\delta \boldsymbol{\omega} \cdot \nabla A) / \rho$ defined solely in terms of the velocity fluctuations, $\boldsymbol{w}$.

In a way similar to that in the SSB, the VGSB framework consists of a set of equations for the fluctuations with respect to a known local equilibrium background (which corresponds to a local approximation of a global equilibrium). Starting from the momentum equation in terms of the fluid velocity $\boldsymbol{v}$ in the frame rotating with $\boldsymbol{\Omega}_{\mathrm{F}}$, we have derived the exact Equation (22) for the velocity $\boldsymbol{w}$ characterizing the departures from the background equilibrium $\boldsymbol{V}(r, z)=$ $r\left[\Omega(r, z)-\Omega_{\mathrm{F}}\right] \hat{\phi}$, Equation 13$)$. In what follows, we derive an evolution equation for the PV-type quantity associated with the velocity fluctuations in an exact way (Equation 156, and then repeat the derivation for the VGSB approximation (Equation 158) with the goal of comparing both results.

Starting from the exact momentum equation (22), taking the curl and using the continuity equation, we arrive to

$$
\begin{aligned}
& \frac{1}{\rho}(\mathcal{D}+\boldsymbol{w} \cdot \nabla) \delta \boldsymbol{\omega}+\left(\frac{\delta \boldsymbol{\omega}}{\rho}\right) \nabla \cdot \boldsymbol{w}+\frac{1}{\rho} \nabla \Omega(r, z) \times \frac{\partial \boldsymbol{w}}{\partial \phi}= \\
& \quad \frac{1}{\rho} \boldsymbol{w}[\nabla \cdot 2 \Omega(r, z) \hat{\boldsymbol{z}}]-\frac{1}{\rho} 2 \Omega(r, z) \hat{\boldsymbol{z}}(\nabla \cdot \boldsymbol{w}) \\
& \quad+\left(\frac{\delta \boldsymbol{\omega}}{\rho} \cdot \nabla\right) \boldsymbol{w}+\left(\frac{2 \Omega(r, z) \hat{\boldsymbol{z}}}{\rho} \cdot \nabla\right) \boldsymbol{w} \\
& \quad-\frac{1}{\rho} \nabla \times\left(w_{z} \frac{\partial V(r, z)}{\partial z} \hat{\boldsymbol{\phi}}+S(r, z) w_{r} \hat{\boldsymbol{\phi}}\right) \\
& \quad+\frac{\nabla P\left(\rho_{h}, e_{h}\right)}{\rho} \times \nabla \frac{1}{\rho_{h}}-\frac{\nabla P}{\rho} \times \nabla \frac{1}{\rho},
\end{aligned}
$$

where it has been natural to define the quantity $\delta \boldsymbol{\omega} \equiv \nabla \times$ $\boldsymbol{w}$, i.e., the vorticity associated with the fluctuations. We can obtain the following relation from the continuity equation (1)

$$
\frac{\delta \boldsymbol{\omega}}{\rho} \nabla \cdot \boldsymbol{w}=-\frac{\delta \boldsymbol{\omega}}{\rho^{2}}(\mathcal{D}+\boldsymbol{w} \cdot \nabla) \rho,
$$

and use it to replace the second term on the left-hand side in Equation (152) to obtain

$$
\begin{aligned}
(\mathcal{D} & +\boldsymbol{w} \cdot \nabla)\left(\frac{\delta \boldsymbol{\omega}}{\rho}\right)+\frac{1}{\rho} \nabla \Omega(r, z) \times \frac{\partial \boldsymbol{w}}{\partial \phi} \\
& +\frac{1}{\rho} \nabla \times\left(w_{z} \frac{\partial V(r, z)}{\partial z} \hat{\boldsymbol{\phi}}+S(r, z) w_{r} \hat{\boldsymbol{\phi}}\right)= \\
& \left(\frac{\delta \boldsymbol{\omega}}{\rho} \cdot \nabla\right) \boldsymbol{w}+\left(\frac{2 \Omega(r, z) \hat{\boldsymbol{z}}}{\rho} \cdot \nabla\right) \boldsymbol{w} \\
& +\frac{\boldsymbol{w}}{\rho}[\nabla \cdot 2 \Omega(r, z) \hat{\boldsymbol{z}}]-\frac{2 \Omega(r, z)}{\rho} \hat{\boldsymbol{z}}(\nabla \cdot \boldsymbol{w}) \\
& +\frac{\nabla P\left(\rho_{h}, e_{h}\right)}{\rho} \times \nabla \frac{1}{\rho_{h}}-\frac{\nabla P}{\rho} \times \nabla \frac{1}{\rho} .
\end{aligned}
$$

If we consider a scalar quantity $A$ which is frozen-in to the flow, i.e., $(\mathcal{D}+\boldsymbol{w} \cdot \nabla) A=0$, we can derive the following identity

$$
\begin{aligned}
\frac{\delta \boldsymbol{\omega}}{\rho} \cdot \nabla[(\mathcal{D}+\boldsymbol{w} \cdot \nabla) A]=\frac{\delta \boldsymbol{\omega}}{\rho} \cdot[(\mathcal{D}+\boldsymbol{w} \cdot \nabla) \nabla A] \\
\quad+\frac{\delta \boldsymbol{\omega}}{\rho} \cdot[\nabla \Omega(r, z)] \partial_{\phi} A+\left[\left(\frac{\delta \boldsymbol{\omega}}{\rho} \cdot \nabla\right) \boldsymbol{w}\right] \cdot \nabla A=0 .
\end{aligned}
$$

We can combine equations (154) and (155) to obtain an evolution equation for $(\delta \omega \cdot \nabla A) / \rho$ as

$$
\begin{aligned}
& (\mathcal{D}+\boldsymbol{w} \cdot \nabla)\left[\frac{\delta \boldsymbol{\omega}}{\rho} \cdot \nabla A\right]+\left[\frac{1}{\rho} \nabla \Omega(r, z) \times \frac{\partial \boldsymbol{w}}{\partial \phi}\right] \cdot \nabla A \\
& +\left[\frac{1}{\rho} \nabla \times\left(w_{z} \frac{\partial V(r, z)}{\partial z} \hat{\boldsymbol{\phi}}+S(r, z) w_{r} \hat{\boldsymbol{\phi}}\right)\right] \cdot \nabla A \\
& =\left[\left(\frac{2 \Omega(r, z) \hat{\boldsymbol{z}}}{\rho} \cdot \nabla\right) \boldsymbol{w}+\frac{\boldsymbol{w}}{\rho}(\nabla \cdot 2 \Omega(r, z) \hat{\boldsymbol{z}})\right. \\
& \left.-\frac{2 \Omega(r, z)}{\rho} \hat{\boldsymbol{z}}(\nabla \cdot \boldsymbol{w})\right] \cdot \nabla A-\left(\frac{\delta \boldsymbol{\omega}}{\rho} \cdot \nabla \Omega(r, z)\right) \frac{\partial A}{\partial \phi} \\
& +\left[-\frac{\nabla \rho_{h} \times \nabla P\left(\rho_{h}, e_{h}\right)}{\rho \rho_{h}^{2}}+\frac{\nabla \rho \times \nabla P}{\rho^{3}}\right] \cdot \nabla A .
\end{aligned}
$$


This result is exact and it follows directly from Equations (1) and $(2)$ in this paper, provided that $A$ is frozen-in to the flow.

Following the same procedures in the VGSB context, starting from the approximate VGSB momentum equation (58) and again taking the curl and combining with the continuity equation we arrive at

$$
\begin{aligned}
\left(\mathcal{D}_{0}\right. & +\boldsymbol{w} \cdot \nabla)\left(\frac{\delta \boldsymbol{\omega}_{0}}{\rho}\right)+\frac{1}{\rho} \nabla\left[V_{0}(z)+S_{0}(z) x\right] \times \frac{\partial \boldsymbol{w}}{\partial y} \\
& +\frac{1}{\rho} \nabla \times\left(w_{z} \frac{\partial V_{0}(z)}{\partial z} \hat{\boldsymbol{y}}+S_{0}(z) w_{x} \hat{\boldsymbol{y}}\right) \\
= & \left(\frac{\delta \boldsymbol{\omega}_{0}}{\rho} \cdot \nabla\right) \boldsymbol{w}+\left(\frac{2 \Omega_{0}(z) \hat{\boldsymbol{z}}}{\rho} \cdot \nabla\right) \boldsymbol{w} \\
& +\frac{1}{\rho} \boldsymbol{w}\left(\nabla \cdot 2 \Omega_{0}(z) \hat{\boldsymbol{z}}\right)-\frac{1}{\rho} 2 \Omega_{0}(z) \hat{\boldsymbol{z}}(\nabla \cdot \boldsymbol{w}) \\
& -\frac{1}{\rho} \nabla P \times \nabla \frac{1}{\rho} .
\end{aligned}
$$

Here, it has also been natural to define $\delta \boldsymbol{\omega}_{0} \equiv \nabla \times \boldsymbol{w}$, i.e., the voticity associated with the fluctuations with respect to the VGSB background. This result is the VGSB equivalent of Equation (154). The notable difference between the VGSB and the exact form is the lack of the $\nabla \rho_{h} \times \nabla P_{h}$ term. Considering a scalar quantity frozen-in to the approximate background $\left(\mathcal{D}_{0}+\boldsymbol{w} \cdot \nabla\right) A=0$, we obtain

$$
\begin{aligned}
\left(\mathcal{D}_{0}\right. & +\boldsymbol{w} \cdot \nabla)\left[\frac{\delta \boldsymbol{\omega}_{0}}{\rho} \cdot \nabla A\right] \\
+ & {\left[\frac{1}{\rho} \nabla\left[V_{0}(z)+S_{0}(z) x\right] \times \frac{\partial \boldsymbol{w}}{\partial y}\right] \cdot \nabla A } \\
& +\left[\frac{1}{\rho} \nabla \times\left(w_{z} \frac{\partial V_{0}(z)}{\partial z} \hat{\boldsymbol{y}}+S_{0}(z) w_{x} \hat{\boldsymbol{y}}\right)\right] \cdot \nabla A \\
= & {\left[\frac{2 \Omega_{0}(z) \hat{\boldsymbol{z}}}{\rho} \cdot \nabla\right) \boldsymbol{w}+\frac{\boldsymbol{w}}{\rho}\left(\nabla \cdot 2 \Omega_{0}(z) \hat{\boldsymbol{z}}\right) } \\
& \left.-\frac{2 \Omega_{0}(z)}{\rho} \hat{\boldsymbol{z}}(\nabla \cdot \boldsymbol{w})\right] \cdot \nabla A \\
& -\left(\frac{\delta \boldsymbol{\omega}_{0}}{\rho} \cdot \nabla\left[V_{0}(z)+x S_{0}(z)\right]\right) \frac{\partial A}{\partial y} \\
& +\left[\frac{\nabla \rho \times \nabla P}{\rho^{3}}\right] \cdot \nabla A .
\end{aligned}
$$

This result is the VGSB version of the exact result in Equation (156). From this form, we can see that in the shearing box (both SSB and VGSB) the lack of the term $\nabla \rho_{h} \times \nabla P_{h}$ prevents the thermodynamic driving of vorticity by the background, which would usually lead in a baroclinic disk to phenomena like the Rossby wave instability (Lovelace et al. 1999). In shearing boxes, this kind of instability can be driven by localized gradients, or for example, by adopting a Boussinesq approximation for the system at the point of Equation (44) and thus retaining the background radial hydrostatic pressure gradient with shear-periodic radial boundary conditions (Lesur \& Papaloizou 2010).
Arlt, R., \& Urpin, V. 2004, A\&A, 426, 755

Bai, X.-N., \& Stone, J. M. 2013, ApJ, 769, 76

Balbus, S. A., \& Hawley, J. F. 1991, ApJ, 3/6, 214

Barker, A. J., \& Latter, H. N. 2015, IMINRAS, 4J0, 21

Blackman, E. G. 2013, European Journal of Physics, 34, 489

Blackman, E. G., \& Pessah, M. E. 2009, ApJL, 704, LII3

Blaes, O., Krolik, J. H., Hirose, S., \& Shabaltas, N. 2011, ApJ, 733, 110

Blandford, R. D., \& Begelman, M. C. 1999, MNRAS, 303, LI

Boyd, J. P. 2000, Chebyshev and Fourier Spectral Methods, 2nd edn. (Dover, New York), online free at http: //www-personal.umich.edu/

jpboyd/BOoK_spectral2000.html

Brandenburg, A., Nordlund, A., Steın, R. F., \& Torkelsson, U. 1995, ApJ, 446,741

Brandenburg, A., Nordlund, A., Stein, R. F., \& Torkelsson, U. 1996, ApJL, 458, L45

Cabot, W. 1996, ApJ, 465, 874

Davis, S. W., Stone, J. M., \& Pessah, M. E. 2010, ApJ, 713, 52

de Avillez, M. A., \& Breitschwerdt, D. 2005, A\&A, 436, ว85

Flock, M., Dzyurkevich, N., Klahr, H., Turner, N. J., \& Henning, T. 2011, ApJ, 735, 122

Fricke, K. 1968, ZAp, 68, 317

Fromang, S., Latter, H., Lesur, G., \& Ogilvie, G. I. 2013, A\&A, 552, A71

Galeev, A. A., Rosner, R., \& Vaiana, G. S. 1979, ApJ, 229, 318

Goldreich, P., \& Lynden-Bell, D. 1965, MNRAS, 130, 125

Goldreich, P., \& Schubert, G. 1967, ApJ, 150, 571

Gressel, O., Elstner, D., Ziegler, U., \& Rudiger, G. 2008, A\&A, 486, L35

Hawley, J. F., Gammie, C. F., \& Balbus, S. A. 1995, ApJ, 440, 142

Hawley, J. F., Gammie, C. F., \& Balbus, S. A. 1996, ApJ, 464, 690

Hawley, J. F., Guan, X., \& Krolik, J. H. 2011, ApJ, '738, 84

Hill, G. W. 1878, American Journal of Mathematics, 1, pp. 5

Ichimaru, S. 1977, ApJ, 214, 840

Johansen, A., \& Levin, Y. ZUU8, A\&A, 490, 501

Joung, M. K. R., \& Mac Low, M.-M. 2006, ApJ, 653, 1266

Kim, W.-T., Ostriker, E. C., \& Stone, J. M. 20U2, ApJ, 581, 1080

Klahr, H. H., \& Bodenheimer, P. 2003, ApJ, 582, 869

Korpi, M. J., Brandenburg, A., Shukurov, A., Tuominen, I., \& Nordlund, Å. 1999, ApJL, 514, L99

Korycansky, D. G., \& Pringle, J. E. 1995, MNRAS, 272, 618

Latter, H. N., Fromang, S., \& Gressel, O. 2010, MNRAS, 406, 848

Lesur, G., Ferreira, J., \& Ogilvie, G. I. 2013, A\&A, J50, A61

Lesur, G., \& Ogilvie, G. I. 2010, MNRAS, 404, L64

Lesur, G., \& Papaloizou, J. C. B. 2010, A\&A, J13, A60

Lin, D. N. C., \& Papaloizou, J. 1980, MNRAS, 191, 31

Lin, M.-K., \& Youdin, A. 2015, ArXiv e-prints, arXiv:1505.02163 [astro-ph.EP]

Lovelace, R. V. E., Li, H., Colgate, S. A., \& Nelson, A. F. 1999, ApJ, 513, 805

Lubow, S. H., \& Pringle, J. E. 1993, ApJ, 409, 360

Lubow, S. H., \& Spruit, H. C. 1995, ApJ, 445, 331

Matsuzaki, T., Matsumoto, R., Tajima, 1., \& Shibata, K. 1997, in

Astronomical Society of the Pacific Conference Series, Vol. 121, IAU

Colloq. 163: Accretion Phenomena and Related Outflows, ed. D. T.

Wickramasinghe, G. V. Bicknell, \& L. Ferrario, 766

Miller, K. A., \& Stone, J. M. 2000, ApJ, 534, 398

Moll, R. 2012, A\&A, 548, A76

Narayan, R., Mahadevan, R., \& Quataert, E. 1998, in Theory of Black Hole Accretion Disks, ed. M. A. Abramowicz, G. Bjornsson, \& J. E. Pringle, 148

Nelson, R. P., Gressel, O., \& Umurhan, O. M. 2013, MNRAS, 435, 2610

Obergaulinger, M., Cerdá-Durán, P., Müller, E., \& Aloy, M. A. 2009, A\&A, 498, 241

Ogilvie, G. I. 2012, MNRAS, 423, 1318

Pedlosky, J. 1982, Geophysical fluid dynamics (Springer-Verlag)

Pessah, M. E., \& Psaltis, D. 2005, ApJ, 628, 879

Piontek, R. A., \& Ostriker, E. C. 2005, ApJ, 629, 849

Proudman, J. 1916, Royal Society of London Proceedings Series A, 92, 408

Rüdiger, G., Arlt, R., \& Shalybkov, D. 2UUL, A\&A, 391, 181

Ryu, D., \& Goodman, J. 1992, ApJ, 388, 438

Spitzer, Jr., L., \& Schwarzschild, M. 1953, ApJ, 118, 106

Stoll, M. H. R., \& Kley, W. 2014, A\&A, 5/2, A/7

Stone, J. M., \& Balbus, S. A. 1996, ApJ, 464, 364

Stone, J. M., Hawley, J. F., Gammie, C. F., \& Balbus, S. A. 1996, ApJ, 463, 656

Suzuki, T. K., \& Inutsuka, S.-i. 2009, ApJL, 691, L49

Suzuki, T. K., \& Inutsuka, S.-i. 2014, ApJ, 184, 121

Suzuki, T. K., Muto, T., \& Inutsuka, S.-1. 2010, ApJ, 718, 1289

Tassoul, J.-L. 2000, Cambridge astrophysics series, vol. 36, Stellar Rotation (Cambridge; New York: Cambridge University Press)

Taylor, G. I. 1917, Royal Society of London Proceedings Series A, 93, 99

Turner, N. J. 2004, ApJL, 6US, L4J

Umurhan. O. M.. Nelson. R. P.. \& Gressel, O. 2015, ArXiv e-prints,

arXiv:1505.01892 [astro-ph.SR]

Umurhan, U. M., \& Regev, U. 2004, A\&A, 427, 855

Urpin, V. 2003, A\&A, 404, 397

Urpin, V., \& Brandenburg, A. 1998, MNRAS, 294, 399

von Zeipel, H. 1924, MNRAS, 84, 665

Wisdom, J., \& Tremaine, S. 1988, AJ, 95, 925 